\title{
Multistep DBT and regular rational extensions of the isotonic oscillator
}

\author{
Yves Grandati \\ Institut de Physique, Equipe BioPhyStat, ICPMB, IF CNRS 2843, \\ Université Paul Verlaine-Metz, 1 Bd Arago, 57078 Metz, Cedex 3, France
}

\begin{abstract}
In some recent articles we developed a new systematic approach to generate solvable rational extensions of primary translationally shape invariant potentials. In this generalized SUSY QM partnership, the DBT are built on the excited states Riccati-Schrödinger (RS) functions regularized via specific discrete symmetries of the considered potential. In the present paper, we prove that this scheme can be extended in a multistep formulation. Applying this scheme to the isotonic oscillator, we obtain new towers of regular rational extensions of this potential which are strictly isospectral to it. We give explicit expressions for their eigenstates which are associated to the recently discovered exceptional Laguerre polynomials and show explicitely that these extensions inherit of the shape invariance properties of the original potential.
\end{abstract}

PACS numbers:

\section{INTRODUCTION}

Since the pionnering work of Gomez-Ullate et al [1], the exceptional orthogonal polynomials (EOP) and their connexion with rational extensions of solvable quantum potentials have been an active research subject [2 20]. The EOP appear to be the constitutive elements of the eigenstates of solvable rational extensions of the second category primary translationally shape-invariant potentials (TSIP) 21 24] In a series of recent papers [18 20] we have developped a new approach which allows to generate infinite sets of such regular solvable extensions starting from every TSIP in a very direct and systematic way without taking recourse to any ansatz or ad hoc deforming functions. This approach is based on a generalization of the usual SUSY partnership built from excited states. The corresponding DarbouxBäcklund Transformations (DBT), which are covariance transformations for the class of Riccati-Schrödinger (RS) equations [21], are based on regularized RS functions corresponding to unphysical (negative energy) eigenfunctions of the initial hamiltonian. They are obtained by using discrete symmetries acting on the parameters of the considered family of potentials. If the use of negative energy states has been already proposed in early years of SUSY MQ development [25], such a systematic scheme has never been envisaged. In the case of the isotonic oscillator, this gives the three infinite sets $L 1, L 2$ and $L 3$ of regular rationally solvable extensions of this potential, the $L 1$ and $L 2$ series potentials being strictly isospectral to the isotonic one and inherits of its shape-invariance property. Very recently Gomez-Ullate, Kamran and Milson [26] introduce a new family of EOP built from the classical Laguerre polynomials via two step Darboux transformations. In the present article we show that the scheme developed in [18 20] admits a very natural n-step extension which allows to recover and generalize the result of [26]. We obtain new infinite towers of rational extensions of the isotonic potential strictly isospectral to this last. Their eigenfunctions are associated to the new EOP families, the one introduced by Gomez-Ullate et al corresponding to the $n=2$ particular case.

The paper is organized as follows. First we recall the basic elements of the method in its one step form and the results obtained for the isotonic system. The regularity of the RS functions is here obtained in a self consistent way, using disconjugacy properties of the Schrödinger equation [27-29] without recourse of the Kienast-Lawton-Hahn theorem [30, 31]. Then we present on a formal level the general $m$-step scheme and in the specific case of the isotonic oscillator, we give a criterion of concrete application in terms of asymptotic behaviour near the origin. Next we consider in a detailed manner the particular $m=2$ case, initially envisaged by Gomez-Ullate et al [26]. We then prove by induction a sufficient condition to be able to build a $m$-step chain of regular and strictly isospectral extended potentials. We finally show that the shape invariance properties of the isotonic potential are hereditary and reached by all these extended potentials.

During the writing of this article, two papers on the same subject appeared. The first one 32] contains results parallels to those developed here. The authors adopt a point of view similar to the one initiated in [18 20] and extended in the present paper. The so-called "virtual states deletion method" corresponding exactly to the use of DBT based on unphysical eigenfunctions associated to regularized RS functions. The second one [33] discusses quite extensively the 2 and 3 steps cases under a slightly different angle and proposes an interesting conjecture on possible "degeneracies" in the set of the new generated ELP. The content of the present article has been established independently of these two works. 


\section{DARBOUX-BÄCKLUND TRANSFORMATIONS (DBT) AND DISCRETE SYMMETRIES}

If $\psi_{\lambda}(x ; a)$ is an eigenstate of $\widehat{H}(a)=-d^{2} / d x^{2}+V(x ; a), a \in \mathbb{R}^{m}, x \in I \subset \mathbb{R}$, associated to the eigenvalue $E_{\lambda}(a)$ $\left(E_{0}(a)=0\right)$

$$
\psi_{\lambda}^{\prime \prime}(x ; a)+\left(E_{\lambda}(a)-V(x ; a)\right) \psi_{\lambda}(x ; a)=0,
$$

then the Riccati-Schrödinger (RS) function $w_{\lambda}(x ; a)=-\psi_{\lambda}^{\prime}(x ; a) / \psi_{\lambda}(x ; a)$ satisfies the corresponding RiccatiSchrödinger (RS) equation [21]

$$
-w_{\lambda}^{\prime}(x ; a)+w_{\lambda}^{2}(x ; a)=V(x ; a)-E_{\lambda}(a) .
$$

It is a well-known fact that the set of general Riccati equations is invariant under the group $\mathcal{G}$ of smooth $S L(2, \mathbb{R})$-valued curves $\operatorname{Map}(\mathbb{R}, S L(2, \mathbb{R}))$ [34, 35]. The particular subclass of Riccati-Schrödinger equations is, as for it, preserved by a specific subset of $\mathcal{G}$. These transformations, called Darboux-Bäcklund Transformations (DBT), are built from any solution $w_{\nu}(x ; a)$ of the initial RS equation $\mathrm{Eq}(2)$ as [21, 34, 35]

$$
w_{\lambda}(x ; a) \stackrel{A\left(w_{\nu}\right)}{\rightarrow} w_{\lambda}^{(\nu)}(x ; a)=-w_{\nu}(x ; a)+\frac{E_{\lambda}(a)-E_{\nu}(a)}{w_{\nu}(x ; a)-w_{\lambda}(x ; a)},
$$

where $E_{\lambda}(a)>E_{\nu}(a) . w_{\lambda}^{(\nu)}$ is then a solution of the RS equation:

$$
-w_{\lambda}^{(\nu) \prime}(x ; a)+\left(w_{\lambda}^{(\nu)}(x ; a)\right)^{2}=V^{(\nu)}(x ; a)-E_{\lambda}(a),
$$

with the same energy $E_{\lambda}(a)$ as in Eq(2) but with a modified potential

$$
V^{(\nu)}(x ; a)=V(x ; a)+2 w_{\nu}^{\prime}(x ; a) .
$$

This can be schematically resumed as

$$
\left\{\begin{array}{l}
w_{\lambda} \stackrel{A\left(w_{\nu}\right)}{\longmapsto} w_{\lambda}^{(\nu)} \\
V \stackrel{A\left(w_{\nu}\right)}{\longmapsto} V^{(\nu)} .
\end{array}\right.
$$

The corresponding eigenstate of $\widehat{H}^{(\nu)}(a)=-d^{2} / d x^{2}+V^{(\nu)}(x ; a)$ can be written

$$
\psi_{\lambda}^{(\nu)}(x ; a)=\exp \left(-\int d x w_{\lambda}^{(\nu)}(x ; a)\right) \sim \frac{1}{\sqrt{E_{\lambda}(a)-E_{\nu}(a)}} \widehat{A}\left(w_{\nu}\right) \psi_{\lambda}(x ; a),
$$

where $\widehat{A}(a)$ is a first order operator given by

$$
\widehat{A}\left(w_{\nu}\right)=d / d x+w_{\nu}(x ; a)
$$

Eq(7) can still be written as

$$
\psi_{\lambda}^{(\nu)}(x ; a) \sim \frac{W\left(\psi_{\nu}, \psi_{\lambda} \mid x\right)}{\psi_{\nu}(x ; a)},
$$

where $W\left(y_{1}, y_{2} \mid x\right)$ is the wronskian of the functions $y_{1}, y_{2}$. 
From $V$, the DBT generates a new potential $V^{(\nu)}$ (quasi) isospectral to the original one and its eigenfunctions are directly obtained from those of $V$ via Eq(7). Nevertheless, in general, $w_{\nu}(x ; a)$ and then the transformed potential $V^{(\nu)}(x ; a)$ are singular at the nodes of $\psi_{\nu}(x ; a)$. For instance, if $\psi_{n}(x ; a)(\nu=n)$ is a bound state of $\widehat{H}(a), V^{(n)}$ is regular only when $n=0$, that is when $\psi_{n=0}$ is the ground state of $\widehat{H}$, and we recover the usual SUSY partnership in quantum mechanics [22, 23].

We can however envisage to use any other regular solution of Eq(2) as long as it has no zero on the considered real interval $I$, even if it does not correspond to a physical state. For some systems, it is possible to obtain such solutions by using specific discrete symmetries $\Gamma_{i}$ which are covariance transformations for the considered family of potentials

$$
\left\{\begin{array}{c}
a \stackrel{\Gamma_{j}}{\rightarrow} a_{i} \\
V(x ; a) \stackrel{\Gamma_{i}}{\rightarrow} V\left(x ; a_{i}\right)=V(x ; a)+U(a) .
\end{array}\right.
$$

$\Gamma_{i}$ acts on the parameters of the potential and transforms the RS function of a physical excited eigenstate $w_{n}$ into a unphysical RS function $v_{n, i}(x ; a)=\Gamma_{i}\left(w_{n}(x ; a)\right)=w_{n}\left(x ; a_{i}\right)$ associated to the negative eigenvalue $\mathcal{E}_{n, i}(a)=$ $\Gamma_{i}\left(E_{n}(a)\right)=U(a)-E_{n}\left(a_{i}\right)<0$.

$$
-v_{n, i}^{\prime}(x ; a)+v_{n, i}^{2}(x ; a)=V(x ; a)-\mathcal{E}_{n, i}(a) .
$$

To $v_{n, i}$ corresponds an unphysical eigenfunction of $\widehat{H}(a)$

$$
\phi_{n, i}(x ; a)=\exp \left(-\int d x v_{n, i}(x ; a)\right)
$$

associated to the eigenvalue $\mathcal{E}_{n, i}(a)$.

If the transformed RS function $v_{n, i}(x ; a)$ of Eq(11) is regular on $I$, it can be used to build a regular extended potential (see $\mathrm{Eq}(5)$ and $\mathrm{Eq}(7 \mathrm{7})$ )

$$
V^{(n, i)}(x ; a)=V(x ; a)+2 v_{n, i}^{\prime}(x ; a)
$$

(quasi)isospectral to $V(x ; a)$. The eigenstates of $V^{(n, i)}$ are given by (see Eq( $(3)$ )

$$
\left\{\begin{array}{c}
w_{k}^{(n, i)}(x ; a)=-v_{n, i}(x ; a)+\frac{E_{k}(a)-\mathcal{E}_{n, i}(a)}{v_{n, i}(x ; a)-w_{k}(x ; a)} \\
\psi_{k}^{(n, i)}(x ; a)=\exp \left(-\int d x w_{k}^{(n, i)}(x ; a)\right) \sim \frac{1}{\sqrt{E_{k}(a)-\mathcal{E}_{n, i}(a)}} \widehat{A}\left(v_{n, i}\right) \psi_{k}(x ; a),
\end{array}\right.
$$

for the respective energies $E_{k}(a)$.

The nature of the isospectrality depends if $1 / \phi_{n, i}(x ; a)$ satisfies or not the appropriate boundary conditions. If it is the case, then $1 / \phi_{n, i}(x ; a)$ is a physical eigenstate of $\widehat{H}^{(n, i)}(a)=-d^{2} / d x^{2}+V^{(n, i)}(x ; a)$ for the eigenvalue $\mathcal{E}_{n, i}(a)$ and we only have quasi-isospectrality between $V(x ; a)$ and $V^{(n, i)}(x ; a)$. If it is not the case, the isospectrality between $V^{(n, i)}(x ; a)$ and $V(x ; a)$ is strict.

The above construction can be summarized by the following diagram

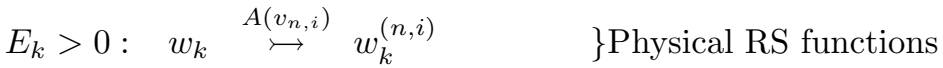

$$
\begin{aligned}
& \Gamma_{j} \downarrow \\
& \mathcal{E}_{k, j}<0: \underbrace{v_{k, j}}_{V} \stackrel{A\left(v_{n, i}\right)}{\longmapsto} \underset{A\left(v_{n, i}\right)}{v_{V^{(n, i)}}^{(n, i)}} \underbrace{\Gamma^{(n, j}}_{\text {Potentials }}\} \text { Regularized (unphysical) RS functions }
\end{aligned}
$$

with

$$
\left\{\begin{array}{c}
\psi_{k}^{(n, i)}=\widehat{A}\left(v_{n, i}\right) \psi_{k} \\
\phi_{k, j}^{(n, i)}=\widehat{A}\left(v_{n, i}\right) \phi_{k, j} .
\end{array}\right.
$$

This procedure can be viewed as a "generalized SUSY QM partnership" where the DBT can be based on excited states RS functions properly regularized by the symmetry $\Gamma_{j}$. 


\section{DISCONJUGACY AND REGULAR EXTENSIONS}

To control the regularity of $v_{n, i}$ we can make use of the disconjugacy properties of the Schrödinger equation for negative eigenvalues.

A second order differential equation like $\mathrm{Eq}(1)$ is said to be disconjugated on $I \subset \mathbb{R}(V(x ; a)$ is supposed to be continuous on $I$ ) if every solution of this equation has at most one zero on $I$ [27, 28]. As it is well known, this zero is necessarily simple and at this value the considered solution changes its sign. For a closed or open interval $I$, the disconjugacy of Eq(1) is equivalent to the existence of solutions of this equation which are everywhere non zero on $I$ [27, 28]. In the following we will consider $I=] 0,+\infty[$.

We have also the following result:

Theorem [27, 28] If there exists a continuously differentiable solution on $I$ of the Riccati inequation

$$
-w^{\prime}(x)+w^{2}(x)+G(x) \leq 0
$$

then the equation

$$
\psi^{\prime \prime}(x)+G(x) \psi(x)=0
$$

is disconjugated on $I$.

In our case, since $\mathcal{E}_{n, i}(a) \leq 0$, we have

$$
-w_{0}^{\prime}(x ; a)+w_{0}^{2}(x ; a)=V(x ; a) \leq V(x ; a)-\mathcal{E}_{n, i}(a),
$$

$w_{0}(x ; a)$ being continuously differentiable on $I$. The above theorem ensure the existence of nodeless solutions $\phi(x ; a)$ of $\mathrm{Eq(1)}$ with $E_{\lambda}(a)=\mathcal{E}_{n, i}(a)$, that is, of regular RS functions $v(x ; a)$ solutions of Eq(11). To prove that a given solution $\phi(x ; a)$ belongs to this category, it is sufficient to determine the signs of the limit values $\phi\left(0^{+} ; a\right)$ and $\phi(+\infty ; a)$. If they are identical then $\phi$ is nodeless and if they are opposite, then $\phi$ presents a unique zero on $I$.

In the first case $V(x ; a)+2 v^{\prime}(x ; a)$ constitutes a regular (quasi)isospectral extension of $V(x ; a)$.

In fact, Bôcher [29] has even established a more precise theorem:

\section{Bôcher's disconjugacy theorem}

If we can find $u$ continuously differentiable on $[a, b]$ such that

$$
u^{\prime}(x)+u^{2}(x)<G(x)
$$

on all $[a, b]$, then nor $\widetilde{\psi}(x)=u(x) \psi(x)-\psi^{\prime}(x)$ nor $\psi(x)$ can have more than one zero in $[a, b]$. Moreover, if $\psi(x)$ vanish in one point of $[a, b]$ then $\widetilde{\psi}(x)$ is everywhere nonzero in this interval (and reciprocally).

If this disconjugacy property is satisfied for every $[a, b] \subset] c, d[$, then it is in fact satisfied in all the open interval ]$c, d[$.

Consider first the case where we have $G(x)>0, \forall x \in] c, d[$. We can then take $u(x)=0$ which implies that any solution $\psi(x)$ of $\mathrm{Eq(18)}$ as well as its derivative $\psi^{\prime}(x)$ have at most one zero in $] c, d\left[\right.$. If $\xi_{0}$ is the corresponding zero of $\psi^{\prime}(x)$ then $\psi(x)$ is strictly monotonous on $] c, \xi_{0}[$ and $] \xi_{0}, d[$.

If we consider now the case of $\mathrm{Eq}(1)$ on $I=] 0,+\infty\left[\right.$ for a strictly negative eigenvalue $\mathcal{E}_{n, i}(a)<0$. From Eq(19) we can take $u(x)=-w_{0}(x ; a)$ to apply Bôcher's theorem from which we deduce that not only Eq(1) is disconjugate on ] $0,+\infty$ [ but also the SUSY partner $\psi^{(0)}(x ; a)$ of any solution of Eq(1) can have at most one zero on ]0, $+\infty[$. Indeed (cf $\mathrm{Eq}(91)$

$$
\widetilde{\psi}(x ; a)=\frac{W\left(\psi_{0}, \psi \mid x\right)}{\psi_{0}(x ; a)}=\psi^{(0)}(x ; a) .
$$




\section{ISOTONIC OSCILLATOR}

Consider the isotonic oscillator potential (ie the radial effective potential for a three dimensional isotropic harmonic oscillator with zero ground-state energy)

$$
V(x ; \omega, a)=\frac{\omega^{2}}{4} x^{2}+\frac{a(a-1)}{x^{2}}+V_{0}(\omega, a), x>0,
$$

with $a=l+1 \geq 1$ and $V_{0}(\omega, a)=-\omega\left(a+\frac{1}{2}\right)$. It is the unique exceptional primary translationally shape invariant potential of the second category [21]. The corresponding Schrödinger equation is the Liouville form of the Laguerre equation on the positive half-line and its physical spectrum, associated to the asymptotic Dirichlet boundary conditions

$$
\psi\left(0^{+} ; \omega, a\right)=0=\psi(+\infty ; \omega, a)
$$

is given by $\left(z=\omega x^{2} / 2, \alpha=a-1 / 2\right)$

$$
E_{n}(a)=2 n \omega, \psi_{n}(x ; \omega, a)=x^{a} e^{-z / 2} L_{n}^{\alpha}(z) .
$$

To $\psi_{n}$ corresponds the RS function [21]

$$
w_{n}(x ; \omega, a)=w_{0}(x ; \omega, a)+R_{n}(x ; \omega, a),
$$

with

$$
w_{0}(x ; \omega, a)=\frac{\omega}{2} x-\frac{a}{x}
$$

and

$$
\begin{aligned}
R_{n}(x ; \omega, a) & =\frac{-2 n \omega}{\omega x-(2 a+1) / x-} ґ \ldots ґ \frac{2(n-j+1) \omega}{\omega x-(2(a+j)-1) / x-} ґ \ldots ґ \frac{2 \omega}{\omega x-(2(a+n)-1) / x} \\
& =\omega x L_{n-1}^{\alpha+1}(z) / L_{n}^{\alpha}(z) .
\end{aligned}
$$

The shape invariance property satisfied by $V$ is $[21-24]$

$$
\widetilde{V}(x ; \omega, a)=V(x ; \omega, a)+2 w_{0}^{\prime}(x ; \omega, a)=V(x ; \omega, a+1)+2 \omega
$$

We have three possible discrete symmetries for $V$ (see $\mathrm{Eq}(10)$ ) which are given by 1)

$$
\omega \stackrel{\Gamma_{+}}{\rightarrow}(-\omega),\left\{\begin{array}{c}
V(x ; \omega, a) \stackrel{\Gamma_{+}}{\rightarrow} V(x ; \omega, a)+\omega(2 a+1) \\
w_{n}(x ; \omega, a) \stackrel{\Gamma_{+}}{\rightarrow} v_{n, 1}(x ; \omega, a)=w_{n}(x ;-\omega, a),
\end{array}\right.
$$

2)

$$
a \stackrel{\Gamma_{\bar{T}}}{\rightarrow} 1-a,\left\{\begin{array}{c}
V(x ; \omega, a) \stackrel{\Gamma_{\bar{T}}}{\rightarrow} V(x ; \omega, a)+\omega(2 a-1) \\
w_{n}(x ; \omega, a) \stackrel{\Gamma_{-}}{\rightarrow} v_{n, 2}(x ; \omega, a)=w_{n}(x ; \omega, 1-a),
\end{array}\right.
$$

3)

$$
(\omega, a) \stackrel{\Gamma_{3}=\Gamma_{+}}{\rightarrow} \Gamma_{-}(-\omega, 1-a)\left\{\begin{array}{c}
V(x ; \omega, a) \stackrel{\Gamma_{3}}{\rightarrow} V(x ; \omega, a)+2 \omega \\
w_{n}(x ; \omega, a) \stackrel{\Gamma_{3}}{\rightarrow} v_{n, 3}(x ; \omega, a)=w_{n}(x ;-\omega, 1-a) .
\end{array}\right.
$$


In the $(\omega, \alpha)$ parameters plane, $\Gamma_{+}$and $\Gamma_{-}$correspond respectively to the reflections with respect to the axes $\omega=0$ and $\alpha=0$. The RS functions $v_{n, i}, i=+,-, 3$, satisfy the respective RS equations

$$
-v_{n, i}^{\prime}(x ; \omega, a)+v_{n, i}^{2}(x ; \omega, a)=V(x ; \omega, a)-\mathcal{E}_{n, i}(\omega, a),
$$

with

$$
\left\{\begin{array}{c}
\mathcal{E}_{n,+}(\omega, a)=E_{-(n+a+1 / 2)}(\omega)<0 \\
\mathcal{E}_{n,-}(\omega, a)=E_{n+1 / 2-a}(\omega) \\
\mathcal{E}_{n, 3}(\omega, a)=E_{-(n+1)}(\omega)<0
\end{array}\right.
$$

These eigenvalues are always negative in the $i=+$ and $i=3$ cases and the inequality $\mathcal{E}_{n,-}(\omega, a) \leq 0$ necessitates to satisfy the constraint $\alpha=a-1 / 2>n$. When $\mathcal{E}_{n, i}(\omega, a) \leq 0$ the Schrödinger equation

$$
\phi^{\prime \prime}(x ; \omega, a)+\left(\mathcal{E}_{n, i}(\omega, a)-V(x ; \omega, a)\right) \phi(x ; \omega, a)=0
$$

is disconjugated on $] 0,+\infty[$. We are then sure that everywhere non zero solutions of Eq(34) exist, the question being to if $\phi_{n, i}(x ; \omega, a)=\exp \left(-\int d x v_{n, i}(x ; \omega, a)\right)$ is such a solution.

\section{L1 AND L2 SERIES OF EXTENSIONS AND CORRESPONDING EXCEPTIONAL LAGUERRE POLYNOMIALS}

The $L 1$ and $L 2$ series of extensions is obtained using the $\Gamma_{+}$and $\Gamma_{-}$symmetries respectively in which case we have synthetically

$$
\left\{\begin{array}{c}
\mathcal{E}_{n, i}(\omega, a)=-2 \omega(a+i(n+1 / 2)) \\
\phi_{n, i}(x ; \omega, a)=\phi_{0, i}(x ; \omega, a) L_{n}^{i \alpha}(-i z)
\end{array}, i= \pm\right.
$$

with

$$
\phi_{0, i}(x ; \omega, a) \sim x^{i \alpha+1 / 2} \exp (i z / 2),
$$

where $\alpha$ is supposed to statisfy the constraint $\alpha>-i n$. The corresponding RS function is given by

$$
v_{n, i}(x ; \omega, a)=v_{0, i}(x ; \omega, a)+Q_{n, i}(x ; \omega, a),
$$

with

$$
v_{0, i}(x ; \omega, a)=-i \frac{\omega}{2} x-\frac{i \alpha+1 / 2}{x}=-\frac{1}{x}(i(z+\alpha)+1 / 2)
$$

and

$$
Q_{n, i}(x ; \omega, a)=-i \omega x L_{n-1}^{i \alpha+1}(-i z) / L_{n}^{i \alpha}(-i z)
$$

Since [30, 31]

$$
\left\{\begin{array}{c}
L_{n}^{\alpha}(x) \underset{x \rightarrow 0^{+}}{\rightarrow} \frac{(\alpha+1)_{n}}{n !}=\left(\begin{array}{c}
n+\alpha \\
n
\end{array}\right) \\
L_{n}^{\alpha}(x) \underset{x \rightarrow+\infty}{\sim} \frac{(-1)^{n}}{n !} x^{n},
\end{array}\right.
$$

where $(X)_{n}=(X) \ldots(X+n-1)$ is the usual Pochhammer symbol [31], we have for $\alpha>1 / 2$

$$
\left\{\begin{array}{c}
\phi_{n,+}(+\infty ; \omega, a)=+\infty \\
\phi_{n,+}\left(0^{+} ; \omega, a\right)=0^{+}
\end{array}\right.
$$


and for $\alpha>n$

$$
\left\{\begin{array}{l}
\phi_{n,+}(+\infty ; \omega, a)=0^{ \pm} \\
\phi_{n,+}\left(0^{+} ; \omega, a\right)= \pm \infty
\end{array}\right.
$$

with $\pm=(-1)^{n}$.

Because of the constraint $\alpha>-i n, \mathrm{Eq}(34)$ is disconjugated both for $i=+$ and $i=-$. Combined to $\mathrm{Eq}(41)$ and $\mathrm{Eq(42)}$, this ensures that $\phi_{n, i}$ keeps a constant strictly positive sign on $] 0,+\infty\left[\right.$ which means that $v_{n, i}(x ; \omega, a)$ is regular on this interval. Note that this result implies in particular that is $L_{n}^{\alpha}(x)$ nodeless on the negative half line and that $L_{n}^{-\alpha}(x)$ has no zero on the positive half line when $\alpha>n$, which is in agreement with the Kienast-Lawton-Hahn theorem [30, 31].

$V^{(n, i)}(x ; \omega, a)=V(x ; \omega, a)+2 v_{n, i}^{\prime}(x ; \omega, a)$ is then a regular extension of $V(x ; \omega, a)$. Since $1 / \phi_{n, i}(x ; \omega, a)$ diverges at the origin, it cannot be a physical eigenstate of $\widehat{H}^{(n, i)}$. Consequently, $V^{(n, i)}$ and $V$ are strictly isospectral.

The (unnormalized) physical eigenstates of $V^{(n, i)}, \psi_{k}^{(n, i)}(x ; \omega, a)=\exp \left(-\int d x w_{k}^{(n, i)}(x ; \omega, a)\right)$, satisfy

$$
\left\{\begin{array}{c}
\widehat{H}^{(n, i)}(\omega, a) \psi_{k}^{(n, i)}(x ; \omega, a)=E_{k}(\omega) \psi_{k}^{(n, i)}(x ; \omega, a) \\
-w_{k}^{(n, i) \prime}(x ; \omega, a)+\left(w_{k}^{(n, i)}(x ; \omega, a)\right)^{2}=V^{(n, i)}(x ; \omega, a)-E_{k}(\omega)
\end{array}\right.
$$

where, using the shape invariance property of $V(\operatorname{cf} \mathrm{Eq}(28))$, we can write

$$
V^{(n, i)}(x ; \omega, a)=V\left(x ; \omega, a_{-1}\right)+2 Q_{n, i}^{\prime}(x ; \omega, a) .
$$

From Eq(14) we have also

$$
\psi_{k}^{(n, i)}(x ; \omega, a)=\widehat{A}\left(v_{n, i}\right) \psi_{k}(x ; \omega, a), k \geq 0 .
$$

More precisely, using the identities [30, 31]

$$
\left\{\begin{array}{c}
L_{n}^{\alpha}(x)+L_{n-1}^{\alpha+1}(x)=L_{n}^{\alpha+1}(x) \\
x L_{n-1}^{\alpha+1}(x)-\alpha L_{n-1}^{\alpha}(x)-n L_{n}^{\alpha-1}(x)=0
\end{array}\right.
$$

we obtain

$$
\begin{aligned}
\psi_{k}^{(n, i)}(x ; \omega, a) & =\left(v_{n, i}(x ; \omega, a)-w_{k}(x ; \omega, a)\right) \psi_{k}(x ; \omega, a) \\
& \sim L_{n, k, \alpha}^{i}(z) \frac{x^{a+i} \exp (-z / 2)}{L_{n}^{i \alpha}(-i z)}
\end{aligned}
$$

where

$$
\left\{\begin{array}{c}
L_{n, k, \alpha}^{+}(z)=L_{n}^{\alpha}(-z) L_{k}^{\alpha+1}(z)+L_{n-1}^{\alpha+1}(-z) L_{k}^{\alpha}(z) \\
L_{n, k, \alpha}^{-}(z)=(k+n+\alpha) L_{k}^{\alpha}(z) L_{n}^{-\alpha}(z)-(-n+\alpha) L_{k}^{\alpha}(z) L_{n-1}^{-\alpha}(z)-(k+\alpha) L_{k-1}^{\alpha}(z) L_{n}^{-\alpha}(z),
\end{array}\right.
$$

are polynomials of degree $n+k$, namely exceptional Laguerre polynomials (ELP) of the $L 1$ and $L 2$ series respectively. From the orthogonality conditions on the eigenstates for fixed values of $n$ and $a$, we retrieve the fact that the ELP $L_{n, k, \alpha}^{i}(z)$ constitute orthogonal families with the corresponding weights

$$
W_{n}^{i}(z)=\frac{z^{a+i / 2} \exp (-z)}{\left(L_{n}^{i \alpha}(-i z)\right)^{2}}
$$




\section{FORMAL SCHEME FOR MULTI-STEP DBT}

\section{A. General elements}

The question of successive iterations of DBT is very natural and is at the center of the construction of the hierarchy of hamiltonians in the usual SUSY QM scheme [41]. Staying at the formal level, it can be simply described by the following straightforward generalization of $\mathrm{Eq}(6)$

$$
\left\{\begin{array}{l}
w_{\lambda} \stackrel{A\left(w_{\nu_{1}}\right)}{\longmapsto} w_{\lambda}^{\left(\nu_{1}\right)} \stackrel{A\left(w_{\nu_{2}}^{\left(\nu_{1}\right)}\right)}{\longmapsto} w_{\lambda}^{\left(\nu_{1}, \nu_{2}\right)} \ldots \stackrel{A\left(w_{\nu_{m}}^{\left(\nu_{1}, \ldots, \nu_{m}-1\right)}\right)}{\longmapsto} w_{\lambda}^{\left(\nu_{1}, \ldots, \nu_{m}\right)} \\
V \stackrel{A\left(w_{\nu_{1}}\right)}{\longmapsto} V^{\left(\nu_{1}\right)} \stackrel{A\left(w_{\nu_{2}}^{\left(\nu_{1}\right)}\right)}{\longmapsto} V^{\left(\nu_{1}, \nu_{2}\right)} \ldots{ }^{A\left(w_{\nu_{m}}^{\left(\nu_{1}, \ldots, \nu_{m-1}\right)}\right)} V^{\left(\nu_{1}, \ldots, \nu_{m}\right)},
\end{array}\right.
$$

where $w_{\lambda}^{\left(\nu_{1}, \ldots, \nu_{m}\right)}$ is a RS function associated to the eigenvalue $E_{\lambda}$ of the potential

$$
V^{\left(\nu_{1}, \ldots, \nu_{m}\right)}(x ; a)=V(x ; a)+2 \sum_{j=1}^{m-1}\left(w_{\nu_{j}}^{\left(\nu_{1}, \ldots, \nu_{j-1}\right)}(x ; a)\right)^{\prime} .
$$

The corresponding eigenfunction is given by (cf Eq(7) and Eq(9) )

$$
\psi_{\lambda}^{\left(\nu_{1}, \ldots, \nu_{m}\right)}(x ; a)=\widehat{A}\left(w_{\nu_{m}}^{\left(\nu_{1}, \ldots, \nu_{m-1}\right)}\right) \psi_{\lambda}^{\left(\nu_{1}, \ldots, \nu_{m-1}\right)}(x ; a)=\widehat{A}\left(w_{\nu_{m}}^{\left(\nu_{1}, \ldots, \nu_{m}-1\right)}\right) \ldots \widehat{A}\left(w_{\nu_{1}}\right) \psi_{\lambda}(x ; a)
$$

that is,

$$
\begin{aligned}
\psi_{\lambda}^{\left(\nu_{1}, \ldots, \nu_{m}\right)}(x ; a) & =\left(w_{\nu_{m}}^{\left(\nu_{1}, \ldots, \nu_{m-1}\right)}(x ; a)-w_{\lambda}^{\left(\nu_{1}, \ldots, \nu_{m-1}\right)}(x ; a)\right) \psi_{\lambda}^{\left(\nu_{1}, \ldots, \nu_{m-1}\right)}(x ; a) \\
& =\frac{W\left(\psi_{\nu_{m}}^{\left(\nu_{1}, \ldots, \nu_{m-1}\right)}, \psi_{\lambda}^{\left(\nu_{1}, \ldots, \nu_{m-1}\right)} \mid x\right)}{\psi_{\nu_{m}}^{\left(\nu_{1}, \ldots, \nu_{m-1}\right)}(x ; a)}
\end{aligned}
$$

where $W\left(y_{1}, \ldots, y_{m} \mid x\right)$ is the wronskian of the functions $y_{1}, \ldots, y_{m}$

$$
W\left(y_{1}, \ldots, y_{m} \mid x\right)=\left|\begin{array}{ccc}
y_{1}(x) & \ldots & y_{m}(x) \\
\ldots & & \ldots \\
y_{1}^{(m-1)}(x) & \ldots & y_{m}^{(m-1)}(x)
\end{array}\right| .
$$

Other useful representations for the extended potentials and of their eigenfunctions are possible. From Sylvester's theorem we can deduce [36] the following property for the Wronskians

$$
W\left(y_{1}, \ldots, y_{m}, y \mid x\right)=\frac{W\left(W\left(y_{1}, \ldots, y_{m}\right), W\left(y_{1}, \ldots, y_{m-1}, y\right) \mid x\right)}{W\left(y_{1}, \ldots, y_{m-1} \mid x\right)} .
$$

Using the identity 36 ]

$$
W\left(u y_{1}, \ldots, u y_{m} \mid x\right)=u^{m} W\left(y_{1}, \ldots, y_{m} \mid x\right)
$$

this gives

$$
\frac{W\left(y_{1}, \ldots, y_{m}, y \mid x\right)}{W\left(y_{1}, \ldots, y_{m} \mid x\right)}=\frac{W\left(\frac{W\left(y_{1}, \ldots, y_{m}\right)}{W\left(y_{1}, \ldots, y_{m-1}\right)}, \frac{W\left(y_{1}, \ldots, y_{m-1}, y\right)}{W\left(y_{1}, \ldots, y_{m-1}\right)} \mid x\right)}{\left.\frac{W\left(y_{1}, \ldots, y_{m} \mid x\right)}{W\left(y_{1}, \ldots, y_{m}-1\right.} \mid x\right)}
$$

and comparing to $\mathrm{Eq}(53)$, we obtain 


$$
\psi_{\lambda}^{\left(\nu_{1}, \ldots, \nu_{m}\right)}(x ; a)=\frac{W\left(\psi_{\nu_{1}}, \ldots, \psi_{\nu_{m}}, \psi_{\lambda} \mid x\right)}{W\left(\psi_{\nu_{1}}, \ldots, \psi_{\nu_{m}} \mid x\right)},
$$

which is the well known Crum formula [37, 40] for the eigenfunctions.

Inserting this result in Eq(51), we then deduce the Crum formula for the potential [37, 40]

$$
V^{\left(\nu_{1}, \ldots, \nu_{m}\right)}(x ; a)=V(x ; a)+2\left(\log W\left(\psi_{\nu_{1}}, \ldots, \psi_{\nu_{m}} \mid x\right)\right)^{\prime \prime} .
$$

Since all the functions $\psi_{\nu_{j}}$ implied in the wronskians in $\mathrm{Eq}(58)$ and $\mathrm{Eq}(59)$ are eigenfunctions of the same hamiltonian $\widehat{H}(a)$, the properties of the determinants allow us to replace in these wronskians the even derivatives $\psi_{\nu_{j}}^{(2 l)}$ by $\left(-E_{\nu_{j}}\right)^{l} \psi_{\nu_{j}}$ and the odd derivatives $\psi_{\nu_{j}}^{(2 l+1)}$ by $\left(-E_{\nu_{j}}\right)^{l} \psi_{\nu_{j}}^{\prime}$.

For instance

$$
\begin{aligned}
W\left(\psi_{\nu_{1}}, \ldots, \psi_{\nu_{2 l}} \mid x\right) & =\left|\begin{array}{ccc}
\psi_{\nu_{1}}(x ; a) & \ldots & \psi_{\nu_{2 l}}(x ; a) \\
\psi_{\nu_{1}}^{\prime}(x ; a) & \ldots & \psi_{\nu_{2 l}}^{\prime}(x ; a) \\
\ldots & \ldots \\
\left(-E_{\nu_{1}}\right)^{l-1} \psi_{\nu_{1}}(x ; a) & \ldots & \left(-E_{\nu_{2 l}}\right)^{l-1} \psi_{\nu_{2 l}}(x ; a) \\
\left(-E_{\nu_{1}}\right)^{l-1} \psi_{\nu_{1}}^{\prime}(x ; a) & \ldots & \left(-E_{\nu_{2 l}}\right)^{l-1} \psi_{\nu_{2 l}}^{\prime}(x ; a)
\end{array}\right| \\
& =\Delta^{\left(\nu_{1}, \ldots, \nu_{2 l}\right)}(x ; a) \prod_{j=1}^{2 l} \psi_{\nu_{j}}(x ; a),
\end{aligned}
$$

where

$$
\Delta^{\left(\nu_{1}, \ldots, \nu_{2 l}\right)}(x ; a)=(-1)^{l}\left|\begin{array}{ccc}
1 & \ldots & 1 \\
w_{\nu_{1}}(x ; a) & \cdots & w_{\nu_{2 l}}(x ; a) \\
\ldots & & \ldots \\
\left(E_{\nu_{1}}\right)^{l-1} & \ldots & \left(E_{\nu_{2 l}}\right)^{l-1} \\
\left(E_{\nu_{1}}\right)^{l-1} w_{\nu_{1}}(x ; a) & \ldots & \left(E_{\nu_{2 l}}\right)^{l-1} w_{\nu_{2 l}}(x ; a)
\end{array}\right|
$$

and

$$
\begin{aligned}
W\left(\psi_{\nu_{1}}, \ldots, \psi_{\nu_{2 l+1}} \mid x\right) & =\left|\begin{array}{ccc}
\psi_{\nu_{1}}(x ; a) & \ldots & \psi_{\nu_{2 l}}(x ; a) \\
\psi_{\nu_{1}}^{\prime}(x ; a) & \ldots & \psi_{\nu_{2 l}}^{\prime}(x ; a) \\
\ldots & \ldots & \ldots \\
\left(-E_{\nu_{1}}\right)^{l-1} \psi_{\nu_{1}}^{\prime}(x ; a) & \ldots & \left(-E_{\nu_{2 l+1}}\right)^{l-1} \psi_{\nu_{2 l+1}}^{\prime}(x ; a) \\
\left(-E_{\nu_{1}}\right)^{l} \psi_{\nu_{1}}(x ; a) & \ldots & \left(-E_{\nu_{2 l+1}}\right)^{l} \psi_{\nu_{2 l+1}}(x ; a)
\end{array}\right| \\
& =\Delta^{\left(\nu_{1}, \ldots, \nu_{2 l+1}\right)}(x ; a) \prod_{j=1}^{2 l+1} \psi_{\nu_{j}}(x ; a),
\end{aligned}
$$

where

$$
\Delta^{\left(\nu_{1}, \ldots, \nu_{2 l+1}\right)}(x ; a)=\left|\begin{array}{ccc}
1 & \ldots & 1 \\
w_{\nu_{1}}(x ; a) & \ldots & w_{\nu_{2 l+1}}(x ; a) \\
\ldots & & \ldots \\
\left(E_{\nu_{1}}\right)^{l-1} w_{\nu_{1}}(x ; a) & \ldots & \left(E_{\nu_{2 l} l}\right)^{l-1} w_{\nu_{2 l+1}}(x ; a) \\
\left(E_{\nu_{1}}\right)^{l} & \ldots & \left(E_{\nu_{2 l}+1}\right)^{l}
\end{array}\right|
$$

Then we obtain the representation of the $\psi_{\lambda}^{\left(\nu_{1}, \ldots, \nu_{m}\right)}$ in terms the so-called Crum-Krein determinants $\Delta^{\left(\nu_{1}, \ldots, \nu_{m}\right)}$ 37, 38, 44]

$$
\psi_{\lambda}^{\left(\nu_{1}, \ldots, \nu_{m}\right)}(x ; a)=\psi_{\lambda}(x ; a) \frac{\Delta^{\left(\nu_{1}, \ldots, \nu_{m}, \lambda\right)}(x ; a)}{\Delta^{\left(\nu_{1}, \ldots, \nu_{m}\right)}(x ; a)} .
$$


Until now, all these results are purely formal. The central problem is now to choose in an appropriate way the family of eigenfunctions $\left(\psi_{\nu_{1}}, \ldots, \psi_{\nu m}\right)$ from which the successive DBT are built (see Eq(58) and Eq(51)) in order to ensure the regularity of the successive extended potentials $V^{\left(\nu_{1}, \ldots, \nu_{m}\right)}$.

A first answer has been given more than half a century ago by Crum [37]. The Crum proposal [37] is to take the successive eigenstates of the discrete bound states spectrum starting from the ground state

$$
\left(\nu_{1}, \ldots, \nu_{m}\right)=(0, \ldots, m-1)
$$

Krein [38, 44, 45] extended this result showing it is possible to choose sets of unnecessarily successive eigenstates if they satisfy a given condition, namely

$$
\left(\nu_{1}, \ldots, \nu_{m}\right)=\left(n_{1}, \ldots, n_{m}\right) \in \mathbb{N}^{m}
$$

with

$$
\left(n-n_{1}\right)\left(n-n_{2}\right) \ldots\left(n-n_{m}\right) \geq 0, \forall n \in \mathbb{N} .
$$

This last is verified if the set $\left(n_{1}, \ldots, n_{m}\right)$ is constituted by "aggregates" of an even number of eigenstates. The possibility to employ sets of two juxtaposed eigenstates has been rediscovered later by Adler [39]. These results have been used extensively in the context of higher order SUSY (see for instance [25, 41 52]). In [48], Samsonov has still extended the Krein-Adler result. Note finally that Fernandez et al [49] employed the specific "Wick rotated" eigenfunctions of negative energies introduced by Shnol' [18, 46, 53] to build successive extensions of the harmonic potential.

\section{B. Application to the isotonic oscillator}

Our aim is to consider the possibility to generalize our construction in a multi-step version on the basis of the regularized RS functions (or unphysical eigenfunctions) obtained above. Staying at a formal level, it would correspond to the following generalization of the diagram Eq(15) (see Eq(50)

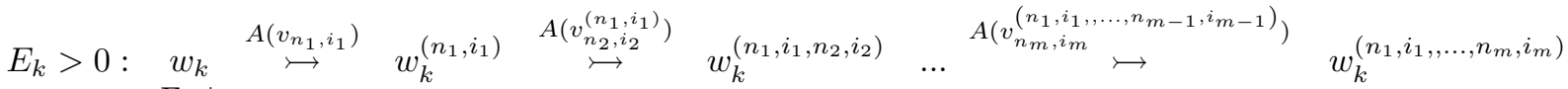

$$
\begin{aligned}
& \Gamma_{i} \downarrow
\end{aligned}
$$

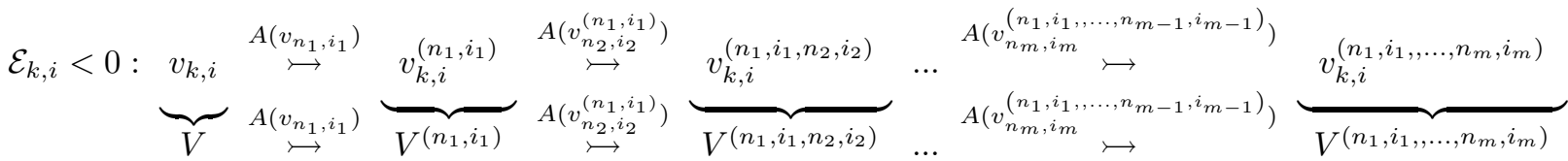

with $v_{k}^{\left(n_{0}\right)}=v_{k}$. Under this way, we generate chains of isospectral extensions $V^{\left(n_{1}, i_{1}, \ldots, n_{m}, i_{m}\right)}$ of the potential $V$ with (see Eq(51) and Eq(59))

$$
\begin{aligned}
V^{\left(n_{1}, i_{1}, \ldots, n_{m}, i_{m}\right)}(x ; a) & =V(x ; a)+2\left(v_{n_{m}, i_{m}}^{\left(n_{1}, i_{1}, \ldots, n_{m-1}, i_{m-1}\right)}(x ; a)\right)^{\prime} \\
& =V(x ; a)+2 \sum_{j=1}^{m}\left(v_{n_{j}, i_{j}}^{\left(n_{1}, i_{1}, \ldots, n_{j-1}, i_{j-1}\right)}(x ; a)\right)^{\prime} \\
& =V(x ; a)+2\left(\log W\left(\phi_{n_{1}, i_{1}}, \ldots, \phi_{n_{m}, i_{m}} \mid x\right)\right)^{\prime \prime}
\end{aligned}
$$

$w_{k}^{\left(n_{1}, i_{1}, \ldots, n_{m}, i_{m}\right)}$ and the $v_{k, i}^{\left(n_{1}, i_{1}, \ldots, n_{m}, i_{m}\right)}$ are RS functions of the extended potential $V^{\left(n_{1}, i_{1}, \ldots, n_{m}, i_{m}\right)}$ associated respectively to the eigenvalues $E_{k}$ and $\mathcal{E}_{k, i}$.

The corresponding eigenfunctions are given by (see Eq(53) and Eq(58)) 


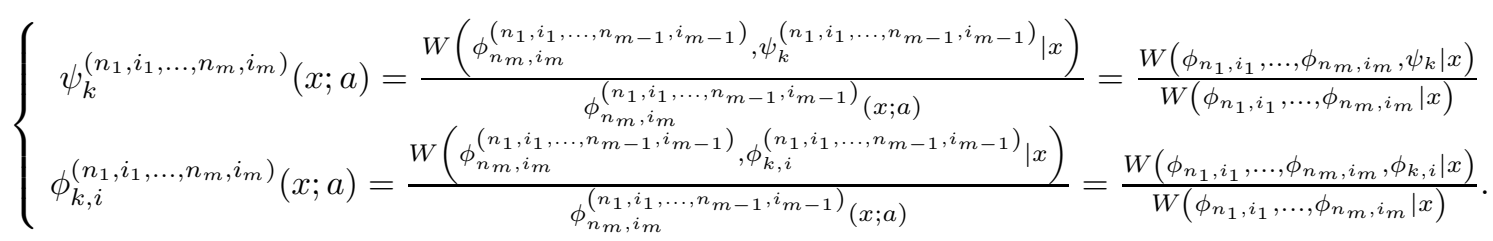

Suppose that at the step $m-1$ all the extended potentials in the chain are regular and strictly isospectral. The problem is now to control the regularity of the potential $V^{\left(n_{1}, i_{1}, \ldots, n_{m}, i_{m}\right)}$ and of its physical eigenstates $\psi_{k}^{\left(n_{1}, i_{1}, \ldots, n_{m-1}, i_{m-1}\right)}$ as well as its strict isospectrality with $V^{\left(n_{1}, i_{1}, \ldots, n_{m-1}, i_{m-1}\right)}$. This is achieved if the unphysical eigenfunction $\phi_{n_{m}, i_{m}}^{\left(n_{1}, i_{1}, \ldots, n_{m-1}, i_{m-1}\right)}$, associated to the DBT $A\left(v_{n_{m}, i_{m}}^{\left(n_{1}, i_{1}, \ldots, n_{m-1}, i_{m-1}\right)}\right)$, is nodeless and if $1 / \phi_{n_{m}, i_{m}}^{\left(n_{1}, i_{1}, \ldots, n_{m-1}, i_{m-1}\right)}$ cannot be into the set of physical eigenstates of $V^{\left(n_{1}, i_{1}, \ldots, n_{m-1}, i_{m-1}\right)}$.

The disconjugacy of the Schrödinger equation

$$
\phi^{\prime \prime}(x ; \omega, a)+\left(\mathcal{E}_{n_{m}, i_{m}}(\omega, a)-V^{\left(n_{1}, i_{1}, \ldots, n_{m-1}, i_{m-1}\right)}(x ; \omega, a)\right) \phi(x ; \omega, a)=0,
$$

for a negative eigenvalue $\mathcal{E}_{n_{m}, i_{m}}$ guarantees that $v_{n_{m}, i_{m}}^{\left(n_{1}, i_{1}, \ldots, n_{m-1}, i_{m-1}\right)}$ is at most singular in one point. The regularity of the extended potential $V^{\left(n_{1}, i_{1}, \ldots, n_{m}, i_{m}\right)}$ and its strict isospectrality with the preceding one in the chain are then satisfied as soon as the unphysical eigenfunction $\phi_{n_{m}, i_{m}}^{\left(n_{1}, i_{1}, \ldots, n_{m-1}, i_{m-1}\right)}$ satisfies appropriate boundary conditions, which was already the argument used above in the one step case. Namely, the strict isospectrality and the regularity are satisfied when $\phi_{n_{m}, i_{m}}^{\left(n_{1}, i_{1}, \ldots, n_{m-1}, i_{m-1}\right)}$ tends to $\infty$ at one extremity of $] 0,+\infty[$ and to 0 at the other extremity, with the same sign for both limits.

In the case of the isotonic oscillator, there are in fact three type of regularization transformations $\Gamma_{i}, i=+,-, 3$ associated respectively to the three series of EOP $L 1, L 2$ and $L 3$ [8, 19]. At each step we can a priori use any of the three type if regularized RS function. However, since the use of DBT associated to RS functions of the L3 series leads only to quasi isospectrality, the argument above is no more valid. Consequently, in the following we exclude this case and consider only DBT built from the RS functions of the $L 1$ or $L 2$ series.

From Eq(64), we see that in the present case

$$
\phi_{n_{m}, i_{m}}^{\left(n_{1}, i_{1}, \ldots, n_{m-1}, i_{m-1}\right)}(x ; \omega, a)=\phi_{0, i_{m}}(x ; \omega, a) R^{\left(n_{1}, i_{1}, \ldots, n_{m}, i_{m}\right)}(x ; \omega, a),
$$

where

$$
R^{\left(n_{1}, i_{1}, \ldots, n_{m}, i_{m}\right)}(x ; \omega, a)=L_{n_{m}}^{i_{m} \alpha}\left(-i_{m} z\right) \frac{\Delta^{\left(n_{1}, i_{1}, \ldots, n_{m}, i_{m}\right)}(x ; \omega, a)}{\Delta^{\left(n_{1}, i_{1}, \ldots, n_{m-1}, i_{m-1}\right)}(x ; \omega, a)}
$$

is a rational function. Due to the presence of the exponential in $\phi_{0, i_{m}}$, the behaviour at infinity of $\phi_{n_{m}, i_{m}}^{\left(n_{1}, i_{1}, \ldots, n_{m-1}, i_{m-1}\right)}$ is the same as for $\phi_{0, i_{m}}$, namely

$$
\left\{\begin{array}{c}
\phi_{n_{m},+}^{\left(n_{1}, i_{1}, \ldots, n_{m-1}, i_{m-1}\right)}(x ; \omega, a) \underset{x \rightarrow+\infty}{\sim} \exp (z / 2) x^{\alpha+1 / 2} R^{\left(n_{1}, i_{1}, \ldots, n_{m},+\right)}(+\infty ; \omega, a) \underset{x \rightarrow+\infty}{\rightarrow} \pm \infty \\
\phi_{n_{m},-}^{\left(n_{1}, i_{1}, \ldots, n_{m-1}, i_{m-1}\right)}(x ; \omega, a) \underset{x \rightarrow+\infty}{\sim} \exp (-z / 2) x^{-\alpha+1 / 2} R^{\left(n_{1}, i_{1}, \ldots, n_{m},-\right)}(+\infty ; \omega, a) \underset{x \rightarrow+\infty}{\rightarrow} 0^{ \pm}
\end{array}\right.
$$

(the sign of the limit being the one of $R^{\left(n_{1}, i_{1}, \ldots, n_{m},+\right)}(+\infty ; \omega, a)$ or $R^{\left(n_{1}, i_{1}, \ldots, n_{m},-\right)}(+\infty ; \omega, a)$ respectively).

The absence of node for $\phi_{n_{m}, i_{m}}^{\left(n_{1}, i_{1}, \ldots, n_{m-1}, i_{m-1}\right)}$ and the strict isospectrality between $V^{\left(n_{1}, i_{1}, \ldots, n_{m-1}, i_{m-1}\right)}$ and $V^{\left(n_{1}, i_{1}, \ldots, n_{m}, i_{m}\right)}$, are simultaneously ensured if and only if we have

$$
\left\{\begin{array}{c}
\phi_{n_{m},+}^{\left(n_{1}, i_{1}, \ldots, n_{m-1}, i_{m-1}\right)}(x ; \omega, a) \underset{x \rightarrow 0^{+}}{\sim} x^{\alpha+1 / 2} R^{\left(n_{1}, i_{1}, \ldots, n_{m},+\right)}\left(0^{+} ; \omega, a\right) \underset{x \rightarrow 0^{+}}{\rightarrow} 0^{ \pm} \\
\phi_{n_{m},-}^{\left(n_{1}, i_{1}, \ldots, n_{m-1}, i_{m-1}\right)}(x ; \omega, a) \underset{x \rightarrow 0^{+}}{\sim} x^{-\alpha+1 / 2} R^{\left(n_{1}, i_{1}, \ldots, n_{m},-\right)}\left(0^{+} ; \omega, a\right) \underset{x \rightarrow 0^{+}}{\rightarrow} \pm \infty,
\end{array}\right.
$$

(the sign of the limit being the one of $R^{\left(n_{1}, i_{1}, \ldots, n_{m},+\right)}\left(0^{+} ; \omega, a\right)$ or $R^{\left(n_{1}, i_{1}, \ldots, n_{m},-\right)}\left(0^{+} ; \omega, a\right)$ respectively), with

$$
\operatorname{sign}\left(R^{\left(n_{1}, i_{1}, \ldots, n_{m}, i_{m}\right)}\left(0^{+} ; \omega, a\right)\right)=\operatorname{sign}\left(R^{\left(n_{1}, i_{1}, \ldots, n_{m}, i_{m}\right)}(+\infty ; \omega, a)\right) .
$$




\section{TWO-STEP CASE}

Before to consider the general case, we first consider the two-step case. We then have to check that the unphysical eigenstates $\phi_{n_{2}, i_{2}}^{\left(n_{1}, i_{1}\right)}$ satisfy the appropriate boundary conditions. Using Eq(35) in Eq(70), we have for $m=2$

$$
\phi_{n_{2}, i_{2}}^{\left(n_{1}, i_{1}\right)}(x ; \omega, a)=\frac{W\left(\phi_{0, i_{1}}(x ; \omega, a) L_{n_{1}}^{i_{1} \alpha}\left(-i_{1} z\right), \phi_{0, i_{2}}(x ; \omega, a) L_{n_{2}}^{i_{2} \alpha}\left(-i_{2} z\right) \mid x\right)}{\phi_{0, i_{1}}(x ; \omega, a) L_{n_{1}}^{i_{1} \alpha}\left(-i_{1} z\right)}
$$

where $\alpha>-i_{j} n_{j}, j=1,2$, or with $\operatorname{Eq(56)~}$

$$
\phi_{n_{2}, i_{2}}^{\left(n_{1}, i_{1}\right)}(x ; \omega, a)=\phi_{0, i_{1}}(x ; \omega, a) \frac{W\left(L_{n_{1}}^{i_{1} \alpha}\left(-i_{1} z\right),\left(x^{\alpha} \exp (z / 2)\right)^{i_{2}-i_{1}} L_{n_{2}}^{i_{2} \alpha}\left(-i_{2} z\right) \mid x\right)}{L_{n_{1}}^{i_{1} \alpha}\left(-i_{1} z\right)} .
$$

If $i_{1}=i_{2}=i$, then

$$
\phi_{n_{2}, i}^{\left(n_{1}, i\right)}(x ; \omega, a)=\phi_{0, i}(x ; \omega, a) \frac{W\left(L_{n_{1}}^{i \alpha}(-i z), L_{n_{2}}^{i \alpha}(-i z) \mid x\right)}{L_{n_{1}}^{i \alpha}(-i z)} .
$$

Using 36]

$$
W\left(y_{1}, \ldots, y_{m} \mid x\right)=\left(\frac{d z}{d x}\right)^{m(m-1) / 2} W\left(y_{1}, \ldots, y_{m} \mid z\right)
$$

and 30,31 ]

$$
\left(L_{n}^{\alpha}(x)\right)^{\prime}=-L_{n-1}^{\alpha+1}(x),
$$

this gives

$$
\phi_{n_{2}, i}^{\left(n_{1}, i\right)}(x ; \omega, a)=\frac{i \omega x^{i \alpha+3 / 2} \exp (i z / 2)}{L_{n_{1}}^{i \alpha}(-i z)}\left|\begin{array}{cc}
L_{n_{1}}^{i \alpha}(-i z) & L_{n_{2}}^{i \alpha}(-i z) \\
L_{n_{1}-1}^{i \alpha+1}(-i z) & L_{n_{2}-1}^{i \alpha+1}(-i z)
\end{array}\right| .
$$

We then have (cf Eq(40))

$$
\left\{\begin{array}{c}
\phi_{n_{2}, i}^{\left(n_{1}, i\right)}(x ; \omega, a) \underset{x \rightarrow 0^{+}}{\sim} \frac{i \omega x^{i \alpha+3 / 2}}{\left(\begin{array}{c}
n_{1}+i \alpha \\
n_{1}
\end{array}\right)}\left|\begin{array}{cc}
\left(\begin{array}{c}
n_{1}+i \alpha \\
n_{1}
\end{array}\right) & \left(\begin{array}{c}
n_{2}+i \alpha \\
n_{2} \\
n_{1}+i \alpha \\
n_{1}-1
\end{array}\right) \\
\left(\begin{array}{c}
n_{2}+i \alpha \\
n_{2}-1
\end{array}\right)
\end{array}\right|=i \omega x^{i \alpha+3 / 2}\left(\begin{array}{c}
n_{2}+i \alpha \\
n_{2}-1
\end{array}\right) \frac{n_{2}-n_{1}}{n_{2}} \\
\phi_{n_{2}, i}^{\left(n_{1}, i\right)}(x ; \omega, a) \underset{x \rightarrow+\infty}{\sim} i^{n_{2}}\left(n_{2}-n_{1}\right) \frac{\omega}{n_{2} !} x^{2 n_{2}+i \alpha-1 / 2} \exp (i z / 2),
\end{array}\right.
$$

that is,

$$
\left\{\begin{array}{c}
\phi_{n_{2},+}^{\left(n_{1},+\right)}(x ; \omega, a) \underset{x \rightarrow 0^{+}}{\sim} \omega x^{(\alpha+2)-1 / 2}\left(\begin{array}{l}
n_{2}+\alpha \\
n_{2}-1
\end{array}\right) \frac{n_{2}-n_{1}}{n_{2}} \underset{x \rightarrow 0^{+}}{\rightarrow} 0^{ \pm} \\
\phi_{n_{2},-}^{\left(n_{1},-\right)}(x ; \omega, a) \underset{x \rightarrow+\infty}{\sim} \frac{\omega\left(n_{2}-n_{1}\right)}{n_{2} !} x^{2 n_{2}+\alpha-1 / 2} \exp (z / 2) \underset{x \rightarrow+\infty}{\rightarrow} \pm \infty
\end{array}, \text { if } a>1\right.
$$

where $\pm=\operatorname{sign}\left(n_{2}-n_{1}\right)$, and

$$
\left\{\begin{array}{c}
\phi_{n_{2},-}^{\left(n_{1},-\right)}(x ; \omega, a) \underset{x \rightarrow 0^{+}}{\sim}(-1)^{n_{2}} \omega x^{-(\alpha-2)-1 / 2}\left(\begin{array}{l}
\alpha-n_{2} \\
n_{2}-1
\end{array}\right) \frac{n_{2}-n_{1}}{n_{2}} \underset{x \rightarrow 0^{+}}{\rightarrow} \pm \infty \\
\phi_{n_{2},-}^{\left(n_{1},-\right)}(x ; \omega, a) \underset{x \rightarrow+\infty}{\sim}(-1)^{n_{2}} \frac{\omega\left(n_{2}-n_{1}\right)}{n_{2} !} x^{2 n_{2}-\alpha-1 / 2} \exp (-z / 2) \underset{x \rightarrow+\infty}{\rightarrow} 0^{ \pm}
\end{array}, \text {if } a>\sup \left(n_{2}, n_{1}\right)\right.
$$

where $\pm=(-1)^{n_{2}} \operatorname{sign}\left(n_{2}-n_{1}\right)$. 
If $i_{1}=-i_{2}=-i$, then using the last of the following identities [54]

$$
\left\{\begin{array}{c}
\left(z^{\alpha} L_{n}^{\alpha}(z)\right)^{\prime}=(n+\alpha) z^{\alpha-1} L_{n}^{\alpha-1}(z) \\
\left(e^{-z} L_{n}^{\alpha}(z)\right)^{\prime}=-e^{-z} L_{n}^{\alpha+1}(z) \\
\left(z^{\alpha} e^{-z} L_{n}^{\alpha}(z)\right)^{\prime}=(n+1) z^{\alpha-1} e^{-z} L_{n+1}^{\alpha-1}(z)
\end{array}\right.
$$

we obtain

$$
\begin{aligned}
\phi_{n_{2}, i}^{\left(n_{1},-i\right)}(x ; \omega, a) & =\omega x^{-i \alpha+3 / 2} \exp (-i z / 2)\left(\frac{2}{\omega}\right)^{i \alpha} \frac{W\left(L_{n_{1}}^{-i \alpha}(i z), z^{i \alpha} \exp (i z) L_{n_{2}}^{i \alpha}(-i z) \mid z\right)}{L_{n_{1}}^{-i \alpha}(i z)} \\
& =\frac{2 x^{i \alpha-1 / 2} \exp (i z / 2)}{L_{n_{1}}^{-i \alpha}(i z)}\left|\begin{array}{cc}
L_{n_{1}}^{-i \alpha}(i z) & z L_{n_{2}}^{i \alpha}(-i z) \\
-i L_{n_{1}-1}^{-i \alpha+1}(i z) & \left(n_{2}+1\right) L_{n_{2}}^{i \alpha-1}(-i z)
\end{array}\right| .
\end{aligned}
$$

We then have (cf Eq(40)

$$
\left\{\begin{array}{c}
\phi_{n_{2}, i}^{\left(n_{1},-i\right)}(x ; \omega, a) \underset{x \rightarrow 0^{+}}{\sim} 2 x^{i \alpha-1 / 2} i^{n_{2}+1} \frac{\left(\alpha+i n_{2}\right) \ldots \alpha}{n_{2} !} \\
\phi_{n_{2}, i}^{\left(n_{1},-i\right)}(x ; \omega, a) \underset{x \rightarrow+\infty}{\sim} i^{n_{2}+1} \frac{1}{n_{2} !}\left(\frac{\omega}{2}\right)^{n_{2}+1} x^{2 n_{2}+i \alpha+3 / 2} \exp (i z / 2) .
\end{array}\right.
$$

Consequently $(a>1)$

$$
\left\{\begin{array}{c}
\phi_{n_{2},+}^{\left(n_{1},-\right)}(x ; \omega, a) \underset{x \rightarrow 0^{+}}{\sim} 2 x^{\alpha-1 / 2} \frac{\left(\alpha+n_{2}\right) \ldots \alpha}{n_{2} !} \underset{x \rightarrow 0^{+}}{\rightarrow} 0^{+} \\
\phi_{n_{2},+}^{\left(n_{1},-\right)}(x ; \omega, a) \underset{x \rightarrow+\infty}{\sim} \frac{1}{n_{2} !}\left(\frac{\omega}{2}\right)^{n_{2}+1} x^{2 n_{2}+a+1} \exp (z / 2) \underset{x \rightarrow+\infty}{\rightarrow}+\infty
\end{array}\right.
$$

and when $\alpha>n_{2}$

$$
\left\{\begin{array}{c}
\phi_{n_{2},-}^{\left(n_{1},+\right)}(x ; \omega, a) \underset{x \rightarrow 0^{+}}{\sim}(-1)^{n_{2}+1} 2 \frac{\left(\alpha-n_{2}\right) \ldots \alpha}{n_{2} !} x^{-\alpha-1 / 2} \underset{x \rightarrow 0^{+}}{\rightarrow} \pm \infty \\
\phi_{n_{2},-}^{\left(n_{1},+\right)}(x ; \omega, a) \underset{x \rightarrow+\infty}{\sim}(-1)^{n_{2}+1} \frac{1}{n_{2} !}\left(\frac{\omega}{2}\right)^{n_{2}+1} x^{2 n_{2}-a+2} \exp (-z / 2) \underset{x \rightarrow+\infty}{\rightarrow} 0^{ \pm},
\end{array}\right.
$$

with $\pm=(-1)^{n_{2}+1}$.

When $a>\sup \left(n_{2}, n_{1}\right)$, we have the correct asymptotic behaviour which ensures the regularity of $V^{\left(n_{1}, i_{1}, n_{2}, i_{2}\right)}(x ; \omega, a)$ and $V^{\left(n_{1}, i_{1}\right)}(x ; \omega, a)$ as their strict isospectrality with $V(x ; \omega, a)$ for every choice of $\left(n_{1}, i_{1}, n_{2}, i_{2}\right)$ with $n_{1} \neq n_{2}$.

We then obtain a chain with two strictly isospectral regular successive extensions of $V$

$$
V \stackrel{A\left(v_{n_{1}, i_{1}}\right)}{\longmapsto} V^{\left(n_{1}, i_{1}\right)} \stackrel{A\left(v_{n_{2}, i_{2}}^{\left(n_{1}, i_{1}\right)}\right)}{\longmapsto} V^{\left(n_{1}, i_{1}, n_{2}, i_{2}\right)}
$$

\section{GENERAL M-STEP CASE}

Consider now the general case of a $m$-step DBT. We proceed by induction, supposing that we have built a chain of $m-1$ regular strictly isospectral potentials

$$
V \stackrel{A\left(v_{n_{1}, i_{1}}\right)}{\longmapsto} V^{\left(n_{1}, i_{1}\right)} \stackrel{A\left(v_{n_{2}, i_{2}}^{\left(n_{1}, i_{1}\right)}\right)}{\longmapsto} \ldots \underbrace{A\left(v_{n_{m}-1, i}^{\left(n_{1}, i_{1}, \ldots, n_{m-2}, i_{m-2}\right)}\right)} V^{\left(n_{1}, i_{1}, \ldots, n_{m-1}, i_{m-1}\right)} .
$$

and that we have $\alpha>-i_{j} n_{j}, \forall j \leq m$. This implies in particular that the $\phi_{n_{j}, i_{j}}^{\left(n_{1}, i_{1}, \ldots, n_{j-1}, i_{j-1}\right)}(x ; \omega, a), j \leq m-1$, which are eigenfunctions of $\widehat{H}^{\left(n_{1}, i_{1}, \ldots, n_{m-1}, i_{m-1}\right)}$ associated to the respective negative eigenvalues $\mathcal{E}_{n_{j-1}, i_{j-1}}(\omega, a)<0$, are all nodeless on $] 0,+\infty[$ and that they keep a constant sign on this interval that we can always take as positive. 
We have to determine what is the constraint to implement for the unphysical eigenfunction $\phi_{n_{m}, i_{m}}^{\left(n_{1}, i_{1}, \ldots, n_{m-1}, i_{m-1}\right)}$ in order that it satisfies the good boundary conditions necessary to build a regular and strictly isospectral extension $V^{\left(n_{1}, i_{1}, \ldots, n_{m}, i_{m}\right)}$ at the next step.

From Eq(74) we know that

$$
\left\{\begin{array}{l}
\phi_{n_{m-1},+}^{\left(n_{1}, i_{1}, \ldots, n_{m-2}, i_{m-2}\right)}(x ; \omega, a) \underset{x \rightarrow+\infty}{\rightarrow}+\infty \\
\phi_{n_{m-1},-}^{\left(n_{1}, i_{1}, \ldots, n_{m-2}, i_{m-2}\right)}(x ; \omega, a) \underset{x \rightarrow+\infty}{\rightarrow} 0^{+}
\end{array}\right.
$$

and consequently we have also (cf Eq(75)

$$
\left\{\begin{array}{c}
\phi_{n_{m-1},+}^{\left(n_{1}, i_{1}, \ldots, n_{m-2}, i_{m-2}\right)}(x ; \omega, a) \underset{x \rightarrow 0^{+}}{\rightarrow} 0^{+} \\
\phi_{n_{m-1},-}^{\left(n_{1}, i_{1}, \ldots, n_{m-2}, i_{m-2}\right)}(x ; \omega, a) \underset{x \rightarrow 0^{+}}{\rightarrow}+\infty
\end{array}\right.
$$

For $x$ sufficiently large or in the vinicity of 0 , we have $\mathcal{E}_{n_{m-1}, \pm}(\omega, a)<V^{\left(n_{1}, i_{1}, \ldots, n_{m-2}, i_{m-2}\right)}(x ; \omega, a)$. The Bôcher disconjugacy theorem then implies that in the neighbourhoods of 0 and of $+\infty$, the functions $\phi_{n_{m-1}, i_{m-1}}^{\left(n_{1}, i_{1}, \ldots, n_{m-2}, i_{m-2}\right)}$ are strictly monotonous, that is strictly decreasing if $i_{m-1}=-$ and strictly increasing if $i_{m-1}=+$.

We already know (cf Eq(74) ) that

$$
\phi_{n_{m}, i_{m}}^{\left(n_{1}, i_{1}, \ldots, n_{m-1}, i_{m-1}\right)}(+\infty ; \omega, a)=\left\{\begin{array}{c} 
\pm \infty, \text { if } i_{m}=+ \\
0^{ \pm}, \text {if } i_{m}=-
\end{array}\right.
$$

We prove now by induction the following result for the unphysical eigenfunctions of the potentials obtained at the $(m-1)^{t h} \operatorname{step}\left(z=\omega x^{2} / 2\right)$

$$
\phi_{n_{m}, i_{m}}^{\left(n_{1}, i_{1}, \ldots, n_{m-1}, i_{m-1}\right)}(x ; \omega, a) \underset{x \rightarrow 0^{+}}{\sim} \widetilde{\phi}_{n_{m}, i_{m}}^{\left(n_{1}, i_{1}, \ldots, n_{m-1}, i_{m-1}\right)}(0) x^{i_{m}\left(\alpha+q\left(i_{1}, \ldots, i_{m}\right)\right)-1 / 2}(1+O(z)),
$$

where $\widetilde{\phi}_{n_{m}, i_{m}}^{\left(n_{1}, i_{1}, \ldots, n_{m-1}, i_{m-1}\right)}(0)$ has the same sign as $\phi_{n_{m}, i_{m}}^{\left(n_{1}, i_{1}, \ldots, n_{m-1}, i_{m-1}\right)}(+\infty ; \omega, a)$ and where $q\left(i_{1}, \ldots, i_{m}\right)=$ $q_{+}\left(i_{1}, \ldots, i_{m}\right)-q_{-}\left(i_{1}, \ldots, i_{m}\right), q_{ \pm}\left(i_{1}, \ldots, i_{m}\right)$ is the number of $i_{j}$ equal to \pm in the set $\left(i_{1}, \ldots, i_{m}\right) . q_{ \pm}\left(i_{1}, \ldots, i_{m}\right)$ can be viewed as the number of state of " \pm charge" used in the chain of DBT at the $m^{\text {th }}$ step and the difference $q\left(i_{1}, \ldots, i_{m}\right)$ as the total charge associated to the chain.

We then have

$$
\left\{\begin{array}{l}
\phi_{n_{m},+}^{\left(n_{1}, i_{1}, \ldots, n_{m-1}, i_{m-1}\right)}(x ; \omega, a) \underset{x \rightarrow 0^{+}}{\rightarrow} 0^{ \pm}, \text {if } a>-q\left(i_{1}, \ldots, i_{m-1},+\right)+1=-q\left(i_{1}, \ldots, i_{m-1}\right) \\
\phi_{n_{m},-}^{\left(n_{1}, i_{1}, \ldots, n_{m-1}, i_{m-1}\right)}(x ; \omega, a) \underset{x \rightarrow 0^{+}}{\rightarrow} \pm \infty, \text { if } a>-q\left(i_{1}, \ldots, i_{m-1},-\right)=1-q\left(i_{1}, \ldots, i_{m-1}\right),
\end{array}\right.
$$

which are necessary conditions in order that the potentials $V^{\left(n_{1}, i_{1}, \ldots, n_{m},+\right)}$ and $V^{\left(n_{1}, i_{1}, \ldots, n_{m},-\right)}$ respectively are regular and strictly isospectral to $V^{\left(n_{1}, i_{1}, \ldots, n_{m-1}, i_{m-1}\right)}$. Note that these constraints are independent of the $n_{j}$ but depend only on the type of the states used to build the chain.

Refering to the results obtained in the 2-step case (see Eq( $\underline{82}$, Eq( $\underline{83}$ ) and Eq(88)), we can verify that this property is valid for $m=2$ since

$$
\left\{\begin{array}{c}
q(+,+)=-q(-,-)=2 \\
q(+,-)=q(-,+)=0
\end{array}\right.
$$

Suppose that Eq(94) is verified till the $(m-1)^{\text {th }}$ step, that is,

$$
\phi_{n, \pm}^{\left(n_{1}, i_{1}, \ldots, n_{m-2}, i_{m-2}\right)}(x ; \omega, a) \underset{x \rightarrow 0^{+}}{\sim} x^{ \pm\left(\alpha+q\left(i_{1}, \ldots, i_{m-2}\right)\right)+1 / 2}(1+O(z))
$$

with $a>-q\left(i_{1}, \ldots, i_{m-2},+\right)=-1-q\left(i_{1}, \ldots, i_{m-2}\right)$, if $i_{m-1}=+$, and $a>1-q\left(i_{1}, \ldots, i_{m-2},-\right)=2-q\left(i_{1}, \ldots, i_{m-2}\right)$, if $i_{m-1}=-$. 
Since $\phi_{n_{m-1}, i_{m-1}}^{\left(n_{1}, i_{1}, \ldots, n_{m-2}, i_{m-2}\right)}$ and $\phi_{k, i}^{\left(n_{1}, i_{1}, \ldots, n_{m-2}, i_{m-2}\right)}$ are both eigenfunctions of $\widehat{H}^{\left(n_{1}, i_{1}, \ldots, n_{m-2}, i_{m-2}\right)}$, using the wronskian theorem [55] in Eq(70) we can write the unphysical eigenfunctions at the $(m-1)^{\text {th }}$ step as

$$
\begin{aligned}
\phi_{k, i}^{\left(n_{1}, i_{1}, \ldots, n_{m-1}, i_{m-1}\right)}(x ; \omega, a)= & \frac{\left(\mathcal{E}_{n_{m-1}, i_{m-1}}-\mathcal{E}_{k, i}\right) \int_{x_{0}}^{x} d \xi \phi_{n_{m-1}, i_{m-1}}^{\left(n_{1}, i_{1}, \ldots, n_{m-2}, i_{m-2}\right)}(\xi ; \omega, a) \phi_{k, i}^{\left(n_{1}, i_{1}, \ldots, n_{m-2}, i_{m-2}\right)}(\xi ; \omega, a)}{\phi_{n_{m-1}, i_{m-1}}^{\left(n_{1}, i_{1}, \ldots, n_{m-2}, i_{m-2}\right)}(x ; \omega, a)}(98) \\
& +\frac{W\left(\phi_{n_{m-1}, i_{m-1}}^{\left(n_{1}, i_{1}, \ldots, n_{m-2}, i_{m-2}\right)}, \phi_{k, i}^{\left(n_{1}, i_{1}, \ldots, n_{m-2}, i_{m-2}\right)} \mid x_{0}\right)}{\phi_{n_{m-1}, i_{m-1}}^{\left(n_{1}, i_{1}, \ldots, n_{m-1}, i_{m-1}\right)}(x ; \omega, a)} .
\end{aligned}
$$

We have to consider several cases corresponding to the four possible couples $\left(i_{m-1}, i\right)$.

1) Consider first the case $i=i_{m-1}=+$ in which we take $x_{0}=0$. Using Eq(92), Eq(98) gives

$$
\phi_{k,+}^{\left(n_{1}, i_{1}, \ldots, n_{m-2}, i_{m-2}, n_{m-1},+\right)}(x ; \omega, a) \sim \frac{\int_{0}^{x} d \xi \phi_{n_{m-1},+}^{\left(n_{1}, i_{1}, \ldots, n_{m-2}, i_{m-2}\right)}(\xi ; \omega, a) \phi_{k,+}^{\left(n_{1}, i_{1}, \ldots, n_{m-2}, i_{m-2}\right)}(\xi ; \omega, a)}{\phi_{n_{m-1},+}^{\left(n_{1}, i_{1}, \ldots, n_{m-2}, i_{m-2}\right)}(x ; \omega, a)} .
$$

Since $\phi_{n_{m-1},+}^{\left(n_{1}, i_{1}, \ldots, n_{m-2}, i_{m-2}\right)}$ and $\phi_{k,+}^{\left(n_{1}, i_{1}, \ldots, n_{m-2}, i_{m-2}\right)}$ are both strictly positive on $] 0,+\infty\left[, \phi_{k,+}^{\left(n_{1}, i_{1}, \ldots, n_{m-2}, i_{m-2}, n_{m-1},+\right)}\right.$ keeps a constant sign on this interval and we can always take it as positive. Using Eq(97), Eq(99) gives

$$
\phi_{k,+}^{\left(n_{1}, i_{1}, \ldots, n_{m-2}, i_{m-2}, n_{m-1},+\right)}(x ; \omega, a) \underset{x \rightarrow 0^{+}}{\sim} x^{\left(\alpha+q\left(i_{1}, \ldots, i_{m-2},+,+\right)\right)-1 / 2}(1+O(z))
$$

Consequently, in this case, Eq(94) is also verified at the $m^{\text {th }}$ step.

2) In the case $i=i_{m-1}=-$, if we take $x_{0}=+\infty$, we have similarly with Eq(91) and Eq(98)

$$
\phi_{k,-}^{\left(n_{1}, i_{1}, \ldots, n_{m-2}, i_{m-2}, n_{m-1},-\right)}(x ; \omega, a) \sim \frac{\int_{x}^{+\infty} d \xi \phi_{n_{m-1},-}^{\left(n_{1}, i_{1}, \ldots, n_{m-2}, i_{m-2}\right)}(\xi ; \omega, a) \phi_{k,-}^{\left(n_{1}, i_{1}, \ldots, n_{m-2}, i_{m-2}\right)}(\xi ; \omega, a)}{\phi_{n_{m-1},-}^{\left(n_{1}, i_{1}, \ldots, n_{m-2}, i_{m-2}\right)}(x ; \omega, a)} .
$$

As in the preceding case, since $\phi_{n_{m-1},-}^{\left(n_{1}, i_{1}, \ldots, n_{m-2}, i_{m-2}\right)}$ and $\phi_{k,-}^{\left(n_{1}, i_{1}, \ldots, n_{m-2}, i_{m-2}\right)}$ are both strictly positive on $] 0,+\infty[$, $\phi_{k,-}^{\left(n_{1}, i_{1}, \ldots, n_{m-2}, i_{m-2}, n_{m-1},-\right)}$ keeps a constant sign on this interval and we can always take it as positive. From Eq(74) we deduce that for any $\varepsilon>0, \int_{\varepsilon}^{+\infty} d \xi \phi_{n_{m-1},-}^{\left(n_{1}, i_{1}, \ldots, n_{m-2}, i_{m-2}\right)}(\xi ; \omega, a) \phi_{k,-}^{\left(n_{1}, i_{1}, \ldots, n_{m-2}, i_{m-2}\right)}(\xi ; \omega, a)$ is finite and positive. Since $\left(a>1-q\left(i_{1}, \ldots, i_{m-2}\right)\right)$

$$
\phi_{n_{m-1},-}^{\left(n_{1}, i_{1}, \ldots, n_{m-2}, i_{m-2}\right)}(x ; \omega, a) \phi_{k,-}^{\left(n_{1}, i_{1}, \ldots, n_{m-2}, i_{m-2}\right)}(x ; \omega, a) \underset{x \rightarrow 0^{+}}{\sim} x^{-2\left(\alpha+q\left(i_{1}, \ldots, i_{m-2}\right)\right)+1}(1+O(z)),
$$

$\mathrm{Eq}(101)$ gives

$$
\phi_{k,-}^{\left(n_{1}, i_{1}, \ldots, n_{m-2}, i_{m-2}, n_{m-1},-\right)}(x ; \omega, a) \underset{x \rightarrow 0^{+}}{\sim} x^{-\left(\alpha+q\left(i_{1}, \ldots, i_{m-2},-,-\right)\right)-1 / 2} .
$$

$\mathrm{Eq}(94)$ is again verified at the $m^{\text {th }}$ step.

3) It remains to consider the cases $i=+=-i_{m-1}$ and $i=-=-i_{m-1}$. Since $\phi_{n,+}^{\left(n_{1}, i_{1}, \ldots, n_{m-2}, i_{m-2}\right)}$ and $\phi_{n,-}^{\left(n_{1}, i_{1}, \ldots, n_{m-2}, i_{m-2}\right)}$ have not the same behavior nor at 0 nor at $+\infty$, Eq(98) is not adapted and we proceed to a direct evaluation of the wronskian $W\left(\phi_{n_{m-1},-}^{\left(n_{1}, i_{1}, \ldots, n_{m-2}, i_{m-2}\right)}, \phi_{k,+}^{\left(n_{1}, i_{1}, \ldots, n_{m-2}, i_{m-2}\right)} \mid x\right)$.

Since $\phi_{n_{m-1},-}^{\left(n_{1}, i_{1}, \ldots, n_{m-2}, i_{m-2}\right)}$ and $\phi_{k,+}^{\left(n_{1}, i_{1}, \ldots, n_{m-2}, i_{m-2}\right)}$ are strictly positive and respectively decreasing and increasing both at infinity and in the vinicity of the origin, $W\left(\phi_{n_{m-1},-}^{\left(n_{1}, i_{1}, \ldots, n_{m-2}, i_{m-2}\right)}, \phi_{k,+}^{\left(n_{1}, i_{1}, \ldots, n_{m-2}, i_{m-2}\right)} \mid x\right)$ has the same positive sign at these two limits. Due to the disconjugacy of

$$
\phi^{\prime \prime}(x ; \omega, a)+\left(\mathcal{E}_{k,+}(\omega, a)-V^{\left(n_{1}, i_{1}, \ldots, n_{m-1}, i_{m-1}\right)}(x ; \omega, a)\right) \phi(x ; \omega, a)=0,
$$


we then deduce that $\phi_{k,+}^{\left(n_{1}, i_{1}, \ldots, n_{m-2}, i_{m-2}, n_{m-1},-\right)}(x ; \omega, a)$ keeps a constant sign (taken as positive) on all the interval ] $0,+\infty[$.

Using (see Eq(97))

$$
\left\{\begin{array}{c}
\phi_{k,+}^{\left(n_{1}, i_{1}, \ldots, n_{m-2}, i_{m-2}\right)}(x ; \omega, a) \underset{x \rightarrow 0^{+}}{\sim} x^{\left(\alpha+q\left(i_{1}, \ldots, i_{m-2}\right)\right)+1 / 2}\left(\widetilde{\phi}_{k,+}^{\left(n_{1}, i_{1}, \ldots, n_{m-2}, i_{m-2}\right)}(0)+O(z)\right) \\
\phi_{n_{m-1},-,}^{\left(n_{1}, i_{1}, \ldots, n_{m-2}, i_{m-2}\right)}(x ; \omega, a) \underset{x \rightarrow 0^{+}}{\sim} x^{-\left(\alpha+q\left(i_{1}, \ldots, i_{m-2}\right)\right)+1 / 2}\left(\widetilde{\phi}_{n_{m-1},-}^{\left(n_{1}, i_{1}, \ldots, n_{m-2}, i_{m-2}\right)}(0)+O(z)\right),
\end{array}\right.
$$

we obtain

$$
\begin{aligned}
& W\left(\phi_{n_{m-1},-}^{\left(n_{1}, i_{1}, \ldots, n_{m-2}, i_{m-2}\right)}, \phi_{k,+}^{\left(n_{1}, i_{1}, \ldots, n_{m-2}, i_{m-2}\right)} \mid x\right) \\
& \underset{x \rightarrow 0^{+}}{\sim} 2\left(\alpha+q\left(i_{1}, \ldots, i_{m-2}\right)\right) \widetilde{\phi}_{k,+}^{\left(n_{1}, i_{1}, \ldots, n_{m-2}, i_{m-2}\right)}(0) \widetilde{\phi}_{n_{m},-}^{\left(n_{1}, i_{1}, \ldots, n_{m-2}, i_{m-2}\right)}(0)+O(z) .
\end{aligned}
$$

Consequently, we deduce

$$
\phi_{k,+}^{\left(n_{1}, i_{1}, \ldots, n_{m-2}, i_{m-2}, n_{m-1},-\right)}(x ; \omega, a) \underset{x \rightarrow 0^{+}}{\sim} x^{\left(\alpha+q\left(i_{1}, \ldots, i_{m-2},-,+\right)\right)-1 / 2}
$$

and

$$
\begin{aligned}
& \phi_{k,-}^{\left(n_{1}, i_{1}, \ldots, n_{m-2}, i_{m-2}, n_{m-1},+\right)}(x ; \omega, a)=\frac{W\left(\phi_{n_{m-1},+}^{\left(n_{1}, i_{1}, \ldots, n_{m-2}, i_{m-2}\right)}, \phi_{k,-}^{\left(n_{1}, i_{1}, \ldots, n_{m-2}, i_{m-2}\right)} \mid x\right)}{\phi_{n_{m},+}^{\left(n_{1}, i_{1}, \ldots, n_{m-2}, i_{m-2}\right)}(x ; \omega, a)} \\
& \underset{x \rightarrow 0^{+}}{\sim}-2\left(\alpha+q\left(i_{1}, \ldots, i_{m-2}\right)\right) \widetilde{\phi}_{k,-}^{\left(n_{1}, i_{1}, \ldots, n_{m-2}, i_{m-2}\right)}(0) x^{-\left(\alpha+q\left(i_{1}, \ldots, i_{m-2}\right)\right)-1 / 2}(1+O(z)) \\
& \underset{x \rightarrow 0^{+}}{\sim} x^{-\left(\alpha+q\left(i_{1}, \ldots, i_{m-2},+,-\right)\right)-1 / 2} .
\end{aligned}
$$

The result Eq(94) is then still valid at the $m^{\text {th }}$ step.

We still have to verify that this condition is compatible with the regularity of the preceding potentials in the chain. For that, note that at the $m^{\text {th }}$ step the explicit expression of the resulting potential and its associated eigenfunctions are a priori independent of the ordering chosen for the sequence. This means that we can consider several chains leading to the same extended potential. Consider the set $\left(n_{1}, i_{1}, \ldots, n_{m-2}, i_{m-2}, n_{m-1}, i_{m-1}\right)$ in which we have regrouped first all the $n_{j}$ associated to a positive $i_{j}$, that is, we have re-arranged the chain as $\left(n_{1}^{\prime}, i_{1}^{\prime}, \ldots, n_{q_{+}}^{\prime}, i_{q_{+}}^{\prime}, n_{q_{+}+1}^{\prime}, i_{q_{+}+1}^{\prime}, \ldots, n_{m-1}^{\prime}, i_{m-1}^{\prime}\right)=\left(n_{1}^{\prime},+, \ldots, n_{q_{+}}^{\prime},+, n_{q_{+}+1}^{\prime},-, \ldots, n_{m-1}^{\prime},-\right)$, where $\left(n_{1}^{\prime}, \ldots, n_{m-1}^{\prime}\right)$ is a permutation of $\left(n_{1}, \ldots, n_{m-1}\right)$ and where $q_{+}=q_{+}\left(i_{1}, \ldots, i_{m-1}\right)$.

Suppose first that $q_{+}=m-1$, that is, $i_{j}^{\prime}=+, \forall j \in\{1, \ldots, m-1\} . \forall j \in\{1, \ldots, m-1\}$ we have $1-q_{+}\left(i_{1}^{\prime}, \ldots, i_{j}^{\prime}\right) \leq 0$ and since $a>1$, the $\phi_{n_{j+1}^{\prime}, i_{j+1}^{\prime}}^{\left(n_{1}^{\prime}, i_{1}^{\prime}, \ldots, n_{j}^{\prime}, i_{j}^{\prime}\right)}$ are all nodeless implying the regularity of all the potentials $V^{\left(n_{1}^{\prime}, i_{1}^{\prime}, \ldots, n_{j}^{\prime}, i_{j}^{\prime}\right)}$ in the chain.

Suppose now that $q_{+}<m-1$, that is, $i_{m-1}^{\prime}=-$. The condition $a>1-q\left(i_{1}^{\prime}, \ldots, i_{m-1}^{\prime}\right)=2-$ $q\left(i_{1}^{\prime}, \ldots, i_{m-2}^{\prime}\right)>1-q\left(i_{1}^{\prime}, \ldots, i_{m-2}^{\prime}\right)$ implies the absence of node for $\phi_{n_{m-1}^{\prime},-}^{\left(n_{1}^{\prime}, i_{1}^{\prime}, \ldots, n_{m-2}^{\prime}, i_{m-2}^{\prime}\right)}$ and consequently the regularity of $V^{\left(n_{1}^{\prime}, i_{1}^{\prime}, \ldots, n_{m-1}^{\prime}, i_{m-1}^{\prime}\right)}$.

We can iterate this reasoning to go backward along the chain and we see clearly that at each step, the opposite of the "total charge" decreases, ensuring the regularity of the associated potential. In this procedure, once we attain the level $q_{+}$, we have eliminated all the negative $i_{j}^{\prime}$ and retrieve then the first case.

Consequently, if we satisfy the condition $a>1-q\left(i_{1}, \ldots, i_{m-1}\right)$ at the step $m$, we are ensured of the existence of a chain of regular extensions which leads to a regular $V^{\left(n_{1}, i_{1}, \ldots, n_{m}, i_{m}\right)}$.

\section{EIGENSTATES OF THE EXTENDED POTENTIALS}

We can generalize the calculations made in the two-step case to determine explicit expressions for the eigenstates of the successive extensions. For that we adopt the ordering mentioned above and consider the spectrum of the potential $V^{\left(n_{1},+, \ldots, n_{q_{+}},+, n_{q_{+}+},-, \ldots, n_{m},-\right)}$ where $q_{+}=q_{+}\left(i_{1}, \ldots, i_{m}\right)$. Using Eq(56) and Eq(178), we have 


$$
\begin{aligned}
& W\left(\phi_{n_{1},+}, \ldots, \phi_{n_{q_{+}},+}, \ldots, \phi_{n_{q_{+}+1},-}, \ldots, \phi_{n_{m},-}, \psi_{k} \mid x\right) \\
\sim & x^{(m+1)(m / 2+a)} e^{-(m+1) z / 2} W\left(e^{z} L_{n_{1}}^{\alpha}(-z), \ldots, e^{z} L_{n_{q_{+}}}^{\alpha}(-z), z^{-\alpha} L_{n_{q_{+}+1}}^{-\alpha}(z), \ldots, z^{-\alpha} L_{n_{m}}^{-\alpha}(z), L_{k}^{\alpha}(z) \mid x\right),
\end{aligned}
$$

that is, with $\mathrm{Eq}(79)$ and $\mathrm{Eq}(84)$

$$
\begin{aligned}
& W\left(\phi_{n_{1},+}, \ldots, \phi_{n_{q_{+}},+}, \ldots, \phi_{n_{q_{+}+},-}, \ldots, \phi_{n_{m},-}, \psi_{k} \mid x\right) \\
\sim & x^{(m+1)(m / 2+a)} e^{\left(q_{+}-(m+1) / 2\right) z} z^{-\left(m-q_{+}\right)(\alpha+m)} \operatorname{det}\left(\Psi_{k}^{\left(n_{1},+, \ldots, n_{q_{+},+, n_{q_{+}+},-, \ldots, n_{m},-}\right)}\right),
\end{aligned}
$$

where $(i, j \in\{1, \ldots, m+1\})$

$$
\left[\Psi_{k}^{\left(n_{1},+, \ldots, n_{q_{+}},+, n_{q_{+}+1},-, \ldots, n_{m},-\right)}\right]_{i, j}=\left\{\begin{array}{c}
L_{n_{j}}^{\alpha+i-1}(-z), \text { if } j \leq q_{+} \\
\left(n_{j}-\alpha-i+2\right)_{i-1} z^{m-i+1} L_{n_{j}}^{-\alpha-i+1}(z), \text { if } q_{+}+1 \leq j \leq m \\
(-1)^{i-1} L_{k-i+1}^{\alpha+i-1}(z), \text { if } j=m+1 .
\end{array}\right.
$$

In the same manner

$$
\begin{aligned}
& W\left(\phi_{n_{1},+}, \ldots, \phi_{n_{q_{+}},+}, \ldots, \phi_{n_{q_{+}+1},-}, \ldots, \phi_{n_{m},-} \mid x\right) \\
\sim & x^{m((m-1) / 2+a)} e^{m z / 2} W\left(L_{n_{1}}^{\alpha}(-z), \ldots, L_{n_{q_{+}}}^{\alpha}(-z), z^{-\alpha} e^{-z} L_{n_{q_{+}+1}}^{-\alpha}(z), \ldots, z^{-\alpha} e^{-z} L_{n_{m}}^{-\alpha}(z) \mid x\right),
\end{aligned}
$$

that is,

$$
\begin{aligned}
& W\left(\phi_{n_{1},+}, \ldots, \phi_{n_{q_{+}},+}, \ldots, \phi_{n_{q_{+}+1},-}, \ldots, \phi_{n_{m},-} \mid x\right) \\
& \sim x^{m((m-1) / 2+a)} z^{\left.\left(m-q_{+}\right)(-\alpha-m+1)\right)} e^{-\left(m / 2-q_{+}\right) z} \operatorname{det}\left(\Phi^{\left(n_{1},+, \ldots, n_{q_{+}},+, n_{q_{+}+1},-, \ldots ., n_{m},-\right)}\right),
\end{aligned}
$$

where $(i, j \in\{1, \ldots, m\})$

$$
\left[\Phi^{\left(n_{1},+, \ldots, n_{q_{+}},+, n_{q_{+}+1},-, \ldots, n_{m},-\right)}\right]_{i, j}=\left\{\begin{array}{c}
L_{n_{j}-i+1}^{\alpha+i-1}(-z), \text { if } j \leq q_{+} \\
\left(n_{j}+1\right)_{i-1} z^{m-i+1} L_{n_{j}+i-1}^{-\alpha-i+1}(z), \text { if } q_{+}+1 \leq j \leq m .
\end{array}\right.
$$

Eq(70) gives then

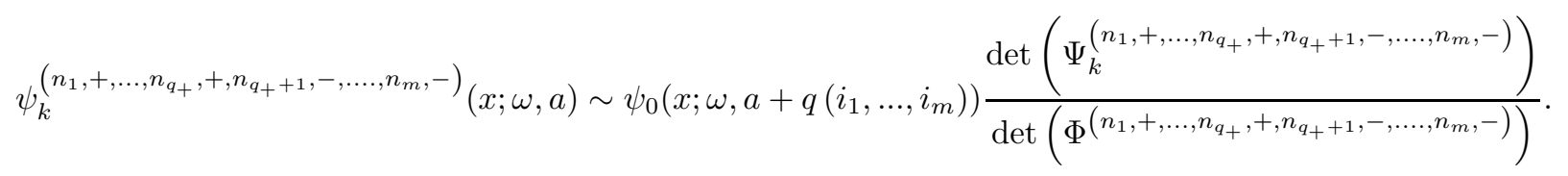

The $\psi_{k}^{\left(n_{1}, i_{1}, \ldots, n_{m}, i_{m}\right)}$ are then obtained as the product of the modified "gauge factor" (ie fundamental state of the initial potential $) \psi_{0}\left(x ; \omega, a+q\left(i_{1}, \ldots, i_{m}\right)\right)$, multiplied by the ratio of two polynomials, the polynomial denominator $\operatorname{det}\left(\Phi^{\left(n_{1},+, \ldots, n_{q_{+}},+, n_{q_{+}+1},-, \ldots, n_{m},-\right)}\right)$ being common to all the eigenstates of the considered extension. The orthogonality condition on the $\psi_{k}^{\left(n_{1}, i_{1}, \ldots, n_{m}, i_{m}\right)}$ ensure that the $\operatorname{det}\left(\Psi_{k}^{\left(n_{1},+, \ldots, n_{q_{+},+, n_{q_{+}+1},-, \ldots ., n_{m},-}\right)}\right)$ constitute an orthogonal family with respect to the weight

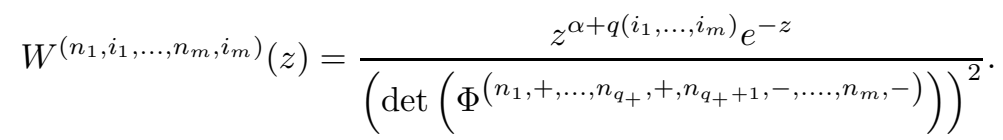




\section{SHAPE INVARIANCE OF THE EXTENDED POTENTIALS}

Consider a chain of regular strictly isospectral extensions

$$
V \stackrel{A\left(v_{n_{1}, i_{1}}\right)}{\longmapsto} V^{\left(n_{1}, i_{1}\right)} \stackrel{A\left(v_{n_{2}, i_{2}}^{\left(n_{1}, i_{1}\right)}\right)}{\longmapsto} \ldots{ }^{A\left(v_{n_{m}, i_{m}}^{\left(n_{1}, i_{1}, \ldots, n_{m-1}, i_{m-1}\right)}\right.} V^{\left(n_{1}, i_{1}, \ldots, n_{m}, i_{m}\right)},
$$

The superpartner of the potential $V^{\left(n_{1}, i_{1}, \ldots, n_{m}, i_{m}\right)}(x ; \omega, a)$ is given by [22, 23]

$$
\widetilde{V}^{\left(n_{1}, i_{1}, \ldots, n_{m}, i_{m}\right)}(x ; \omega, a)=V^{\left(n_{1}, i_{1}, \ldots, n_{m}, i_{m}\right)}(x ; \omega, a)+2\left(w_{0}^{\left(n_{1}, i_{1}, \ldots, n_{m}, i_{m}\right)}(x ; \omega, a)\right)^{\prime},
$$

$w_{0}^{\left(n_{1}, i_{1}, \ldots, n_{m}, i_{m}\right)}(x ; \omega, a)$ being the RS function associated to the ground level of $V^{\left(n_{1}, i_{1}, \ldots, n_{m}, i_{m}\right)}\left(E_{0}(\omega)=0\right)$.

Since (see Eq(3))

$$
w_{0}^{\left(n_{1}, i_{1}, \ldots, n_{m}, i_{m}\right)}(x ; \omega, a)=-v_{n_{m}, i_{m}}^{\left(n_{1}, i_{1}, \ldots, n_{m-1}, i_{m-1}\right)}(x ; \omega, a)-\frac{\mathcal{E}_{n_{m}, i_{m}}(\omega, a)}{v_{n_{m}, i_{m}}^{\left(n_{1}, i_{1}, \ldots, n_{m-1}, i_{m-1}\right)}(x ; \omega, a)-w_{0}^{\left(n_{1}, i_{1}, \ldots, n_{m-1}, i_{m-1}\right)}(x ; \omega, a)}
$$

we have with $\mathrm{Eq(69)}$

$$
\begin{aligned}
\widetilde{V}^{\left(n_{1}, i_{1}, \ldots, n_{m}, i_{m}\right)}(x ; \omega, a)= & V^{\left(n_{1}, i_{1}, \ldots, n_{m-1}, i_{m-1}\right)}(x ; \omega, a) \\
& -2\left(\frac{\mathcal{E}_{n_{m}, i_{m}}(\omega, a)}{v_{n_{m}, i_{m}}^{\left(n_{1}, i_{1}, \ldots, n_{m-1}, i_{m-1}\right)}(x ; \omega, a)-w_{0}^{\left(n_{1}, i_{1}, \ldots, n_{m-1}, i_{m-1}\right)}(x ; \omega, a)}\right)^{\prime}
\end{aligned}
$$

We proceed by induction. Suppose that the potential $V^{\left(n_{1}, i_{1}, \ldots, n_{m-1}, i_{m-1}\right)}$ has the same shape invariance properties than $V$

$$
\begin{aligned}
\widetilde{V}^{\left(n_{1}, i_{1}, \ldots, n_{m-1}, i_{m-1}\right)}(x ; \omega, a) & =V^{\left(n_{1}, i_{1}, \ldots, n_{m-1}, i_{m-1}\right)}(x ; \omega, a)+2\left(w_{0}^{\left(n_{1}, i_{1}, \ldots, n_{m-1}, i_{m-1}\right)}(x ; \omega, a)\right)^{\prime} \\
& =V^{\left(n_{1}, i_{1}, \ldots, n_{m-1}, i_{m-1}\right)}(x ; \omega, a+1)+2 \omega .
\end{aligned}
$$

As shown in [19], this is effectively the case for $m=2$. It results

$$
\widetilde{V}^{\left(n_{1}, i_{1}, \ldots, n_{m}, i_{m}\right)}(x ; \omega, a)=V^{\left(n_{1}, i_{1}, \ldots, n_{m}, i_{m}\right)}(x ; \omega, a+1)+2 \omega-2\left(\Delta^{\left(n_{1}, i_{1}, \ldots, n_{m}, i_{m}\right)}(x ; \omega, a)\right)^{\prime}
$$

where

$$
\begin{aligned}
\Delta^{\left(n_{1}, i_{1}, \ldots, n_{m}, i_{m}\right)}(x ; \omega, a)= & \frac{\mathcal{E}_{n_{m}, i_{m}}(\omega, a)}{v_{n_{m}, i_{m}}^{\left(n_{1}, i_{1}, \ldots, n_{m-1}, i_{m-1}\right)}(x ; \omega, a)-w_{0}^{\left(n_{1}, i_{1}, \ldots, n_{m-1}, i_{m-1}\right)}(x ; \omega, a)} \\
& +w_{0}^{\left(n_{1}, i_{1}, \ldots, n_{m-1}, i_{m-1}\right)}(x ; \omega, a)+v_{n_{m}, i_{m}}^{\left(n_{1}, i_{1}, \ldots, n_{m-1}, i_{m-1}\right)}(x ; \omega, a+1) .
\end{aligned}
$$

Suppose also that

$$
\Delta^{\left(n_{1}, i_{1}, \ldots, n_{m-1}, i_{m-1}\right)}(x ; \omega, a)=0
$$

which again is verified for $m=2$ [19]. We can then write

$$
\begin{aligned}
v_{n_{m-1}, i_{m-1}}^{\left(n_{1}, i_{1}, \ldots, n_{m-2}, i_{m-2}\right)}(x ; \omega, a+1)= & -w_{0}^{\left(n_{1}, i_{1}, \ldots, n_{m-2}, i_{m-2}\right)}(x ; \omega, a) \\
& -\frac{\mathcal{E}_{n_{m-1}, i_{m-1}}(\omega, a)}{v_{n_{m-1}, i_{m-1}}^{\left(n_{1}, i_{1}, \ldots, n_{m-2}, i_{m-2}\right)}(x ; \omega, a)-w_{0}^{\left(n_{1}, i_{1}, \ldots, n_{m-2}, i_{m-2}\right)}(x ; \omega, a)}
\end{aligned}
$$


Moreover we have

$$
\left\{\begin{array}{l}
w_{0}^{\left(n_{1}, i_{1}, \ldots, n_{m-1}, i_{m-1}\right)}(x ; \omega, a)=-v_{n_{m-1}, i_{m-1}}^{\left(n_{1}, i_{1}, \ldots, n_{m-2}, i_{m-2}\right)}(x ; \omega, a)-\frac{\mathcal{E}_{n_{m-1}, i_{m-1}}(\omega, a)}{v_{n_{m-1}, i_{m-1}}^{\left(n_{1}, i_{1}, \ldots, n_{m-2}, i_{m-2}\right)}(x ; \omega, a)-w_{0}^{\left(n_{1}, i_{1}, \ldots, n_{m-2}, i_{m-2}\right)}(x ; \omega, a)} \\
v_{n_{m}, i_{m}}^{\left(n_{1}, i_{1}, \ldots, n_{m-1}, i_{m-1}\right)}(x ; \omega, a)=-v_{n_{m-1}, i_{m-1}}^{\left(n_{1}, i_{1}, \ldots, n_{m-2}, i_{m-2}\right)}(x ; \omega, a)+\frac{\mathcal{E}_{n_{m}, i_{m}}(\omega, a)-\mathcal{E}_{n_{m-1}}, i_{m-1}(\omega, a)}{v_{n_{m-1}, i_{m-1}}^{\left(n_{1}, i_{1}, \ldots, n_{m-2}, i_{m-2}\right)}(x ; \omega, a)-v_{n_{m}, i_{m}}^{\left(n_{1}, i_{1}, \ldots, n_{m-2}, i_{m-2}\right)}(x ; \omega, a)}
\end{array}\right.
$$

and (see $\operatorname{Eq}(\underline{33}))$

$$
\mathcal{E}_{n_{m}, i_{m}}(\omega, a+1)-\mathcal{E}_{n_{m-1}, i_{m-1}}(\omega, a+1)=\mathcal{E}_{n_{m}, i_{m}}(\omega, a)-\mathcal{E}_{n_{m-1}, i_{m-1}}(\omega, a) .
$$

Inserting $\mathrm{Eq}(\underline{124}), \mathrm{Eq}(123)$ and $\mathrm{Eq}(125)$ into $\mathrm{Eq(121)}$, we obtain after a little elementary algebra

$$
\Delta^{\left(n_{1}, i_{1}, \ldots, n_{m}, i_{m}\right)}(x ; \omega, a)=0 .
$$

By induction, this property is verified for every $m$. Then Eq(120) becomes

$$
\widetilde{V}^{\left(n_{1}, i_{1}, \ldots, n_{m}, i_{m}\right)}(x ; \omega, a)=\widetilde{V}^{\left(n_{1}, i_{1}, \ldots, n_{m}, i_{m}\right)}(x ; \omega, a+1)+2 \omega
$$

that is, the potential has also the same shape invariance properties than the isotonic potential. The translational shape invariance of the isotonic potential is then hereditary in such chain of extensions.

\section{CONCLUSION}

We have shown that the "generalized SUSY QM partnership" that we have previously elaborated in a one-step scheme can be extended in a multi-step formulation. We have proven the necessary conditions to obtain chains of regular extensions of the isotonic potential of arbitrary length and have given explicit expressions for their eigenstates. We also established explicitely the hereditary character of the shape invariance properties of the isotonic potential which are common to all the potentials in a given chain.

The case of the generic potentials of the second category of primary TSIP [21], namely Pöschl-Teller or Scarf potentials, can be considered in the same way. This work is in progress and a forthcoming paper is in preparation. For the first category exceptional TSIP, due to the strict isospectrality constraint, the only interesting case in which we can envisage such chain of extensions is the ERKC potential [20]. This is the object of further investigations.

\section{ACKNOWLEDGMENTS}

I would like to thank A. Bérard, R. Milson and C. Quesne for stimulating exchanges and very interesting suggestions.

[1] D. Gómez-Ullate, N. Kamran and R. Milson, "The Darboux transformation and algebraic deformations of shape invariant potentials", J. Phys. A 37, 1789-1804 (2004).

[2] D. Gómez-Ullate, N. Kamran and R. Milson, "Supersymmetry and algebraic Darboux transformations", J. Phys. A 37, 10065-10078 (2004).

[3] D. Gómez-Ullate, N. Kamran, and R. Milson, "An extended class of orthogonal polynomials defined by a Sturm-Liouville problem", J. Math. Anal. Appl. 359, 352 (2009).

[4] D. Gómez-Ullate, N. Kamran and R. Milson, "An extension of Bochner's problem: exceptional invariant subspaces", J. Approx. Theory 162, 987-1006 (2010).

[5] D.Gómez-Ullate, N. Kamran and R. Milson, "Exceptional orthogonal polynomials and the Darboux transformation", J. Phys. A 43434016 (2010).

[6] D. Gómez-Ullate, N. Kamran and R. Milson, "On orthogonal polynomials spanning a non-standard flag", arXiv:1101.5584 (2011).

[7] C. Quesne, "Exceptional orthogonal polynomials, exactly solvable potentials and supersymmetry", J. Phys. A 41, 392001 (2008). 
[8] C. Quesne, "Solvable rational potentials and exceptional orthogonal polynomials in supersymmetric quantum mechanics", SIGMA 5, 084, $24 \mathrm{p}(2009)$.

[9] B. Bagchi, C. Quesne and R. Roychoudhury, "Isospectrality of conventional and new extended potentials, second-order supersymmetry and role of $\mathcal{P} \mathcal{T}$ symmetry", Pramana J. Phys. 73 337-347 (2009).

[10] B. Bagchi and C. Quesne, "An update on $\mathcal{P} \mathcal{T}$-symmetric complexified Scarf II potential, spectral singularities and some remarks on the rationally-extended supersymmetric partners", J. Phys. A 43305301 (2010).

[11] S. Odake and R. Sasaki, "Infinitely many shape invariant potentials and new orthogonal polynomials", Phys. Lett. B 679, $414-417$ (2009).

[12] S. Odake and R. Sasaki, "Another set of infinitely many exceptional $\left(\mathrm{X}_{l}\right)$ Laguerre polynomials", Phys. Lett. B 684, 173-176 (2009).

[13] C-L. Ho, S. Odake and R. Sasaki, "Properties of the exceptional (X $)_{l}$ Laguerre and Jacobi polynomials," YITP-09-70, arXiv :0912.5477[math-ph] (2009).

[14] S.Odake and R. Sasaki, "Infinitely many shape invariant potentials and cubic identities of the Laguerre and Jacobi polynomials", J. Math. Phys. 51, 053513 (2010).

[15] R. Sasaki, S. Tsujimoto and A. Zhedanov, "Exceptional Laguerre and Jacobi polynomials and the corresponding potentials through Darboux-Crum transformations", J. Phys. A 43315204 (2010).

[16] D. Dutta and P. Roy, "Conditionally exactly solvable potentials and exceptional orthogonal polynomials", J. Math. Phys. 51, 042101 (2010).

[17] C-L. Ho and R. Sasaki, "Zeros of the exceptional Laguerre and Jacobi polynomials," arXiv:1102.5669 [math-ph] (2011).

[18] Y. Grandati and A. Bérard, "Solvable rational extension of translationally shape invariant potentials", arXiv:0912.3061 (2009), to appear in the proceedings of the Jairo Charris Seminar 2010, "Algebraic Aspects of Darboux Transformations, Quantum Integrable Systems and Supersymmetric Quantum Mechanics".

[19] Y. Grandati, "Solvable rational extensions of the isotonic oscillator", Ann. Phys. 326, 2074-2090 (2011).

[20] Y. Grandati, "Solvable rational extensions of the Morse and Kepler-Coulomb potentials", J. Math. Phys. 52, 103505 (2011).

[21] Y. Grandati and A. Bérard, "Rational solutions for the Riccati-Schrödinger equations associated to translationally shape invariant potentials", Ann. Phys. 325, 1235-1259 (2010).

[22] F. Cooper, A. Khare and U. Sukhatme, Supersymmetry in Quantum Mechanics (World Scientific, Singapore, 2001).

[23] R. Dutt, A. Khare and U. P. Sukhatme, "Supersymmetry, shape invariance and exactly solvable potentials," Am. J. Phys. 56, 163-168 (1988).

[24] L. Gendenshtein, "Derivation of exact spectra of the Schrodinger equation by means of supersymmetry," JETP Lett. 38, 356-359 (1983).

[25] C. V. Sukumar, "Supersymmetric quantum mechanics of one-dimensional systems", J. Phys. A, 18, 2917-2936 (1985).

[26] D. Gómez-Ullate, N. Kamran and R. Milson, "Two-step Darboux transformations and exceptional Laguerre polynomials ", J. Math. Anal. Appl. 387 410-418 (2012).

[27] P. Hartman, Ordinary differential equations, (John Wiley, New York, 1964).

[28] W. A. Coppel, Disconjugacy, (Springer, Berlin, 1971).

[29] M. Bôcher, Leçons sur les méthodes de Sturm, p.51-52 (Gauthier-Villars, Paris, 1917).

[30] G. Szegö, Orthogonal polynomials, (American Mathematical Society, Providence, 1975).

[31] A. Erdélyi, W. Magnus, F. Oberhettinger and F. G. Tricomi, Higher transcendental functions (Mc Graw-Hill, New York, 1953).

[32] S. Odake and R. Sasaki, " Exactly Solvable Quantum Mechanics and Infinite Families of Multi-indexed Orthogonal Polynomials ", Phys. Lett. B 702, 164-170 (2011).

[33] C. Quesne, "Higher-order SUSY, exactly solvable potentials, and exceptional orthogonal polynomials", Mod. Phys. Lett. A 26 1843-1852 (2011).

[34] J. F. Cariñena and A. Ramos, "Integrability of Riccati equation from a group theoretical viewpoint," Int. J. Mod. Phys. A, 14, 1935-1951 (1999).

[35] J. F. Cariñena, A. Ramos and D. J. Fernandez, "Group theoretical approach to the intertwined hamiltonians", Ann. Phys., 292, 42-66 (2001).

[36] T. Muir (revised and enlarged by W.H. Metzler), A treatise on the theory of determinants (Dover, New York, 1960).

[37] M. M. Crum, "Associated Sturm-Liouville systems", Q. J. Math., 6, 121-127 (1955).

[38] M. G. Krein, "On a continuous analogue of a Christoffel formula from the theory of orthogonal polynomials", Dokl. Akad. Nauk SSSR, 113, 970 (1957).

[39] V. E. Adler, "A modification of Crum method", Theor. Math. Phys., 101 1381-1386 (1994).

[40] V.B. Matveev and M.A. Salle, Darboux Transformations and Solitons (Springer-Verlag, Berlin, 1991).

[41] C. V. Sukumar, "Supersymmetry, factorization of the Schrödinger equation and an hamiltonian hierarchy", J. Phys. A, 18, L57-L61 (1985).

[42] A. A. Andrianov, N. V. Borisov and M. V. Ioffe, "The factorization method and quantum systems with equivalent energy spectra ", Phys. Lett. A 105, 19-22 (1985).

[43] A. A. Andrianov, M. V. Ioffe and V. P. Spiridonov, "Higher-derivative supersymmetry and the Witten index", Phys. Lett. A 174, 273-279 (1993).

[44] V. G. Bagrov and B. F. Samsonov, "Darboux transformation, factorization, and supersymmetry in one-dimensional quantum mechanics", Theor. Math. Phys. 104 356-367 (1995).

[45] V. G. Bagrov and B. F. Samsonov, "Darboux transformation and elementary exact solutions of the Schrödinger equation", 
Pramana J. Phys. 49 563-580 (1997).

[46] B.F. Samsonov and I.N. Ovcharov, "Darboux transformation and exactly solvable potentials with quasi-equidistant spectrum", Russ. Phys. J. 38, 765-771 (1995).

[47] V. G. Bagrov, B. F. Samsonov and L. A. Shekoyan, "N-order Darboux transformation and a spectral problem on semiaxis", arXiv:9804.032 (1998).

[48] B.F. Samsonov, "New possibilities for supersymmetry breakdown in quantum mechanics and second order irreducible Darboux transformations", Phys. Lett. A 263, 274-280 (1999).

[49] D. J. Fernández, V. Hussin and B. Mielnik, "A simple generation of exactly solvable anharmonic oscillators", Phys. Lett. A 244, 309-316 (1998).

[50] D. J. Fernández, J. Negro and L. M. Nieto, "Second-order supersymmetric periodic potentials", Phys. Lett. A 275, 338-349 (2000).

[51] D. J. Fernández, R. Muñoz and A. Ramos, "Second order SUSY transformations with 'complex energies"”, Phys. Lett. A 308, 11-16 (2003).

[52] B. Mielnik, L. M. Nieto and O. Rosas-Ortiz, "The finite difference algorithm for higher order supersymmetry", Phys. Lett. A 269, 70-78 (2000).

[53] E. E. Shnol', Appendix B in: S. Dubov, V. M. Eleonskii and N. E. Kulagin, "Equidistant spectra of anharmonic oscillators", Chaos 4, 47 -53 (1994).

[54] G. E. Andrews, R. Askey and R. Roy, Special functions (Cambridge University Press, Cambridge, 2000).

[55] A. Messiah, Mécanique quantique T1 (Dunod, Paris, 1969). 